\title{
Study of a Fractional-Order Chaotic System Represented by the Caputo Operator
}

\author{
Ndolane Sene (iD \\ Département de Mathématiques de la Décision, Université Cheikh Anta Diop de Dakar, \\ Faculté des Sciences Economiques et Gestion, BP 5683 Dakar Fann, Senegal \\ Correspondence should be addressed to Ndolane Sene; ndolanesene@yahoo.fr \\ Received 19 January 2021; Revised 5 April 2021; Accepted 3 June 2021; Published 14 June 2021 \\ Academic Editor: Guillermo Huerta Cuellar \\ Copyright (c) 2021 Ndolane Sene. This is an open access article distributed under the Creative Commons Attribution License, \\ which permits unrestricted use, distribution, and reproduction in any medium, provided the original work is properly cited. \\ This paper is presented on the theory and applications of the fractional-order chaotic system described by the Caputo fractional \\ derivative. Considering the new fractional model, it is important to establish the presence or absence of chaotic behaviors. The \\ Lyapunov exponents in the fractional context will be our fundamental tool to arrive at our conclusions. The variations of the \\ model's parameters will generate chaotic behavior, in general, which will be established using the Lyapunov exponents and \\ bifurcation diagrams. For the system's phase portrait, we will present and apply an interesting fractional numerical discretization. \\ For confirmation of the results provided in this paper, the circuit schematic is drawn and simulated. As it will be observed, the \\ results obtained after the simulation of the numerical scheme and with the Multisim are in good agreement.
}

\section{Introduction}

In the last decade, chaos theory has attracted many researchers. Chaos theory is a field of mathematics that can be applied in many domains: from modeling chaotic financial systems [1], in representing circuit schematics [2, 3], and others. The chaotic systems and their circuit schematics have received many investigations in the literature, see, for example, [2-4]. With the new development of fractional calculus, some researchers were interested in modeling chaotic systems using the new and old fractional operators. The impact of the fractional derivatives is an interesting question which merits investigations. Many problems are opened in fractional calculus. The first question is how to construct the numerical scheme to obtain the phase portraits of chaotic systems. Many numerical techniques are used in fractional differential equations that can be applied naturally to solve chaotic systems. In [5], Danca finds interesting remarks that the characterization of the hyperchaotic system using two positive Lyapunov exponents is not an adequate definition in a fractional context. Therefore, modeling chaos using fractional operators is an open problem that admits some interesting interrogations which are not solved yet.
Fractional calculus finds many recent advancements in fractional operators and their applicability in real-world problems. For recent advances of fractional calculus and its applications, see the following investigations [1, 6-10].

The chaos literature in integer version and fractional context is very long, but we recall the investigations we find essential for our study. In [11], Pacheco et al. study a new analysis of a new chaotic system with different families of hidden and self-excited attractors by using the fractional operator. In [12], Rajagopal et al. analyze using fractionalorder derivative a memristor no equilibrium chaotic system; they proposed synchronizing their introduced model. In other words, they try to find adequate control to synchronize slave and master chaotic systems. In [2], Avalos-Ruiz et al. propose a control implementation on an FPGA for a class of fractional chaotic systems in the context of fractional variable order derivative with Mittag-Leffler kernel. In [13], Perez et al. propose the fractional-order chaotic system in the context of conformable Liouville-Caputo operator. At the same time, they present a novel numerical scheme for the used fractional operator to depict the phase portraits of their proposed chaotic system. In [14], Gomez et al. investigate the chaos in a calcium oscillation model using the Mittag-Leffler 
fractional derivative operator. In [15], Atangana and Gomez offer a new hyperchaotic system in the context of the MittagLeffler fractional operator. They provided a numerical scheme to depict the considered model's phase portrait in the fractional operators' context. They mainly apply their numerical procedure to the fractional Chua model. In [16], $\mathrm{Li}$ et al. introduce a new four-wing model with integer-order derivative, which will be subject to investigations in the fractional context. In [3], Akgul gives a chaotic system for modeling the memcapacitor model using a fractional-order derivative. In [17], Rajagopal et al. continue their exciting investigation of the fractional-order chaotic system in the fractional calculus field. They propose a work on the multiscroll chaotic system and give an application to the synchronization of their proposed model. In [18], Akgul et al. address a user interface for the generation of random numbers based on fractional and integer-order chaotic systems. For more investigations in chaos, see in the following investigations $[4,12-14,19]$.

The novelties of the present paper are described in the following lines. This paper focuses on the phase space, the bifurcation diagrams, and the chaos's characterization using the Lyapunov exponents for a fractional version of the $\mathrm{Li}$ et al. chaotic system. We analyze the impact of the fractionalorder derivative on the dynamics of a fractional version of the system in [16] with Lyapunov exponents and bifurcation diagram aids. Its possible implementation in terms of electrical modeling has been proposed as well. One of the novelties of the present work is the Lyapunov exponents in the fractional context, which is an open problem. Note that the Lyapunov exponents' properties in the integer context for hyperchaotic and chaotic behaviors are not adequate definitions in the fractional version. This remark was first discussed in [5]. Furthermore, the slight variation of the model's parameters in the dynamics has been investigated using bifurcation diagrams. We provide in this paper that the initial conditions impact the chaotic behaviors in the fractional context. The circuit schematic of the fractional chaotic system and the results after simulation in Multisim confirm the theoretical findings obtained via the numerical scheme.

The paper is structured in the following form. In Section 2 , we define the fractional operators' necessaries for our work. In Section 3, we present the fractional-order chaotic system described by the Caputo derivative. Section 4 offers the numerical scheme used to obtain the considered fractional system's phase portraits. In Section 5, we illustrate the numerical method by depicting the supposed system's phase portraits with different fractional-order of the Caputo derivative. We also characterize the nature of the chaos using the Lyapunov exponents. In Section 6, we present the bifurcation diagrams and phase space according to the variation of the considered fractional-order chaotic system's parameters. In Section 7, we give the possible implementation of the fractional-order chaotic system in modeling electrical circuits; this section will confirm the paper's theoretical findings. Section 8 provides the stability analysis of the fractional-order chaotic system's equilibrium points with the Matignon criterion used in fractional calculus. In
Section 9, we analyze the sensitivity of the initial conditions in the fractional-order chaotic system. We finish with the final remarks and future perspectives of research.

\section{On Fractional Operators in Fractional Calculus}

In this paper, we study a fractional differential system; before, it was indispensable to recall the fractional operators used in this work. Many fractional derivatives exist, those with singularities as the Caputo and Riemann-Liouville derivatives, those with nonsingularities as the exponential derivative, and the Mittag-Leffler fractional derivative. Due to space limitations, we provide the Caputo derivative and the Riemann-Liouville derivative and their generalizations.

Definition 1 (see [20, 21]). The integral defined by Riemann and Liouville called Riemann-Liouville integral of a given function $z:[0,+\infty[\longrightarrow \mathbb{R}$ is

$$
\left(I^{\alpha} z\right)(t)=\frac{1}{\Gamma(\alpha)} \int_{0}^{t}(t-s)^{\alpha-1} z(s) \mathrm{d} s,
$$

with the function $\Gamma(\ldots)$ symbolizing the Gamma Euler function and under the order $\alpha>0$.

There exists a generalization of the Riemann-Liouville operator recently proposed in the literature; we have the following definition.

Definition 2 (see [22]). The generalized Riemann-Liouville fractional integral of a given function $z:[0,+\infty[\longrightarrow \mathbb{R}$ is

$$
I^{\alpha, \rho} z(t)=\frac{1}{\Gamma(\alpha)} \int_{0}^{t}\left(\frac{t^{\rho}-s^{\rho}}{\rho}\right)^{\alpha-1} z(s) \frac{\mathrm{d} s}{s^{1-\rho}},
$$

where the orders $\alpha$ and $\rho$ satisfy the following relationship $\alpha$ and $\rho>0$ and the function gamma is $\Gamma(\ldots)$, for all $t>0$.

We can observe that when the order $\rho=1$, we get the classical Riemann-Liouville integral operator. Furthermore, when $\alpha=\rho=1$, we have the integer version of the integral.

Caputo proposed another derivative due to the inconvenience of the Riemann-Liouville operator. We recall the Caputo derivative in the following definitions and its generalization.

Definition 3 (see [20, 21]). The Caputo fractional derivative of order $\alpha \in(0,1)$ can be represented as the form of the function $z:[0,+\infty[\longrightarrow \mathbb{R}:$

$$
D_{c}^{\alpha} z(t)=\frac{1}{\Gamma(1-\alpha)} \int_{0}^{t}(t-s)^{-\alpha} z^{\prime}(s) \mathrm{d} s,
$$

with the Gamma Euler function denoted by $\Gamma(\ldots)$.

Definition 4 (see [22]). The generalized Caputo derivative of order $\alpha \in(0,1)$ is described as the form of the function $z:[0,+\infty[\longrightarrow \mathbb{R}:$ 


$$
D^{\alpha, \rho} z(t)=\frac{1}{\Gamma(1-\alpha)} \int_{0}^{t}\left(\frac{t^{\rho}-s^{\rho}}{\rho}\right)^{-\alpha} z^{\prime}(s) \mathrm{d} s,
$$

with the orders $\alpha$ and $\rho$ satisfying the relations $\alpha \in(0.1)$ and $\rho>0$ and the Gamma function is denoted by $\Gamma(\ldots)$, for all $t>0$.

There exist many fractional operators, and all of them have their advantages. The use of the Caputo derivative in the present work has many motivations. Firstly, all fractional operators take into account the memory effect. Secondly, our main motivation is the initial conditions because the Riemann-Liouville derivative cannot consider them. In the Riemann-Liouville derivative case, the starting condition should be an integral form known in physics to be unrealistic. It cannot be satisfied in many real-world problems. Another basic motivation is in the context of Riemann-Liouville; the derivative of a constant is not null, contrary to the context of the Caputo derivative where the derivative of a constant is null.

\section{Fractional-Order Chaotic System}

This section provides a fractional version of the chaotic model based on the system presented by Li et al. [16]. Before the investigation, we recall $\mathrm{Li}$ et al. chaotic equations represented with the integer-order derivative by the following equation:

$$
\begin{aligned}
& x^{\prime}=a x+y+y z, \\
& y^{\prime}=-x z+y z, \\
& z^{\prime}=-b z-c x y+m,
\end{aligned}
$$

with initial conditions

$$
\begin{aligned}
& x(0)=1, \\
& y(0)=-1, \\
& z(0)=1 .
\end{aligned}
$$

The strange attractor is obtained at $a=1, b=1$, and $m=$ 1 according to Li et al. work. The Lyapunov exponents of the model presented in system (5) are $\mathrm{LYE}_{1}=0.409, \mathrm{LYE}_{2}=0$, and $\mathrm{LYE}_{3}=-1.773$. In the original paper, the system presented in equation (5) is called a four-wing chaotic equation and admits three real equilibrium points, which are unstable and have two other complex equilibrium points. For more explanations related to the system in equation (5), see [16]. In our present work, we consider the memory effect generated by the fractional operators. Therefore, we investigate the following modified fractional-order chaotic system:

$$
\begin{aligned}
& D_{c}^{\kappa} x=a x+y+y z, \\
& D_{c}^{\beta} y=-x z+y z, \\
& D_{c}^{\alpha} z=-b z-c x y+m,
\end{aligned}
$$

with the initial condition defined by the following relation:

$$
\begin{aligned}
& x(0)=x_{0}=0.2, \\
& y(0)=y_{0}=0.2, \\
& z(0)=z_{0}=0.2 .
\end{aligned}
$$

Our objective is to give a new fractional-order system; therefore, we set the following values for the parameters of model (7); there are $a=1, b=1, c=1$, and $m=0$. In terms of comparison, we can observe that equation (7) is a fractional version of equation (5). Furthermore, we can observe when the order of the Caputo derivative is $\kappa=\beta=\alpha=1$ in equation (7); we recover the constructive equation of original equation (5) presented in [16]. The difference is in the initial conditions. This paper will be to focus on detecting the chaotic behavior when the fractional-order derivative is utilized. The fractional-order operator impact will be analyzed and proved by the comparison of the attractors' geometries. The variation of the model's parameters such as $a$ and $c$ generate strange actuators and will be confirmed using the bifurcation diagrams. We will also establish via the stability analysis of the equilibrium points of our considered chaotic system; there exists a chaotic region according to the variation of the order of the Caputo derivative. Equation (7) is said the system with commensurate fractional-order when $\kappa=\beta=\alpha$; else, it is said the equation with incommensurate fractional-order.

\section{Numerical Scheme for the Fractional-Order Chaotic System}

In this section, we apply the numerical scheme proposed by Garrapa [23] for our proposed fractional-order equation (7) necessary to depict the phase portraits. This numerical discretization uses the numerical approach of the Riemann-Liouville integral. In this section, we consider equation (7) with commensurate fractional-order. The first step will be to determine the analytical solution of fractional differential equation (7) using Riemann-Liouville integral:

$$
\begin{aligned}
& x(t)=x(0)+I^{\alpha} u\left(t, x_{1}\right), \\
& y(t)=y(0)+I^{\alpha} v\left(t, x_{1}\right), \\
& z(t)=z(0)+I^{\alpha} w\left(t, x_{1}\right) .
\end{aligned}
$$

We set the following function obtained from model (7):

$$
\begin{gathered}
u\left(t, x_{1}\right)=a x+y+y z, \\
v\left(t, x_{1}\right)=-x z+y z, \\
w\left(t, x_{1}\right)=-b z-c x y .
\end{gathered}
$$

For the graphical representations, we introduce the discretization at the point $t_{n}$; then, equations (9)-(11) can be written in the following form:

$$
\begin{aligned}
& x\left(t_{n}\right)=x(0)+I^{\alpha} u\left(t_{n}, x_{1}\right), \\
& y\left(t_{n}\right)=y(0)+I^{\alpha} v\left(t_{n}, x_{1}\right),
\end{aligned}
$$




$$
z\left(t_{n}\right)=z(0)+I^{\alpha} w\left(t_{n}, x_{1}\right)
$$

Introducing the step-size $h$ and $t_{n}=n h$, the discretization of the fractional integrals can be represented for the functions $u, v$, and $w$ as the following:

$$
\begin{aligned}
& I^{\alpha} u\left(t_{n}, x_{1}\right)=h^{\alpha} \sum_{j=1}^{n} d_{n-j} u\left(t_{j}, x_{1 j}\right), \\
& I^{\alpha} v\left(t_{n}, x_{1}\right)=h^{\alpha} \sum_{j=1}^{n} d_{n-j} v\left(t_{j}, x_{1 j}\right), \\
& I^{\alpha} w\left(t_{n}, x_{1}\right)=h^{\alpha} \sum_{j=1}^{n} d_{n-j} w\left(t_{j}, x_{1 j}\right),
\end{aligned}
$$

where the parameters are given by

$$
\begin{aligned}
d_{n-j} & =\frac{\left((n-j+1)^{\alpha}-(n-j)^{\alpha}\right)}{1 /(\Gamma(1+\alpha))} \\
d_{n} & =\frac{\left((n+1)^{\alpha}-(n)^{\alpha}\right)}{1 /(\Gamma(1+\alpha))}
\end{aligned}
$$

For the simplification of the numerical scheme of the Riemann-Liouville integral, we introduce the first-order interpolant polynomial of the functions $u(\tau), v(\tau)$, and $w(\tau)$ given by the following relations [23]:

$$
u(\tau)=u\left(t_{j+1}, x_{1 j+1}\right)+\frac{\tau-t_{j+1}}{h}\left[u\left(t_{j+1}, x_{1 j+1}\right)-u\left(t_{j}, x_{1 j}\right)\right]
$$

$$
v(\tau)=v\left(t_{j+1}, x_{1 j+1}\right)+\frac{\tau-t_{j+1}}{h}\left[v\left(t_{j+1}, x_{1 j+1}\right)-v\left(t_{j}, x_{1 j}\right)\right],
$$

$w(\tau)=w\left(t_{j+1}, x_{1 j+1}\right)+\frac{\tau-t_{j+1}}{h}\left[w\left(t_{j+1}, x_{1 j+1}\right)-w\left(t_{j}, x_{1 j}\right)\right]$.

Replacing equations (20)-(22) into equations (16)-(18), we get, after recursive summation, more simple formulas for the numerical approach of the fractional integrals:

$$
\begin{aligned}
& I^{\alpha} u\left(t_{n}, x_{1}\right)=h^{\alpha}\left[\bar{d}_{n}^{(\alpha)} u(0)+\sum_{j=1}^{n} d_{n-j}^{(\alpha)} u\left(t_{j}, x_{1 j}\right)\right], \\
& I^{\alpha} v\left(t_{n}, x_{1}\right)=h^{\alpha}\left[\bar{d}_{n}^{(\alpha)} v(0)+\sum_{j=1}^{n} d_{n-j}^{(\alpha)} v\left(t_{j}, x_{1 j}\right)\right], \\
& I^{\alpha} w\left(t_{n}, x_{1}\right)=h^{\alpha}\left[\bar{d}_{n}^{(\alpha)} w(0)+\sum_{j=1}^{n} d_{n-j}^{(\alpha)} w\left(t_{j}, x_{1 j}\right)\right],
\end{aligned}
$$

where the parameters are given explicitly as the following form:

$$
\bar{d}_{n}^{(\alpha)}=\frac{(n-1)^{\alpha}-n^{\alpha}(n-\alpha-1)}{\Gamma(2+\alpha)},
$$

and furthermore, when $n=1,2, \ldots$, then the parameter $d$ can be expressed as the following forms:

$$
\begin{aligned}
& d_{0}^{(\alpha)}=\frac{1}{\Gamma(2+\alpha)} \\
& d_{n}^{(\alpha)}=\frac{(n-1)^{\alpha+1}-2 n^{\alpha+1}+(n+1)^{\alpha+1}}{\Gamma(2+\alpha)} .
\end{aligned}
$$

For the numerical schemes which are used to represent the graphics, it is obtained after plugging equations (23)-(27) into equations (13)-(15); we have the following expressions:

$$
\begin{aligned}
& x\left(t_{n}\right)=x(0)+h^{\alpha}\left[\bar{d}_{n}^{(\alpha)} u(0)+\sum_{j=1}^{n} \kappa_{n-j}^{(\alpha)} u\left(t_{j}, x_{1 j}\right)\right], \\
& y\left(t_{n}\right)=y(0)+h^{\alpha}\left[\bar{d}_{n}^{(\alpha)} v(0)+\sum_{j=1}^{n} \kappa_{n-j}^{(\alpha)} v\left(t_{j}, x_{1 j}\right)\right], \\
& z\left(t_{n}\right)=z(0)+h^{\alpha}\left[\bar{d}_{n}^{(\alpha)} w(0)+\sum_{j=1}^{n} \kappa_{n-j}^{(\alpha)} w\left(t_{j}, x_{1 j}\right)\right] .
\end{aligned}
$$

For the previous representations, it is necessary to recall the numerical discretization for our functions $u, v$, and $w$; we have the following:

$$
\begin{gathered}
u\left(t_{j}, x_{1 j}\right)=a x_{j}+y_{j}+y_{j} z_{j}, \\
v\left(t_{j}, x_{1 j}\right)=-x_{j} z_{j}+y_{j} z_{j}, \\
w\left(t_{j}, x_{1 j}\right)=-b z_{j}-c x_{j} y_{j} .
\end{gathered}
$$

The convergence and stability are not detailed in this section, see [23]; here, we just give information related to these two properties. We assume that $x\left(t_{n}\right), y\left(t_{n}\right)$, and $z\left(t_{n}\right)$ are the approximate numerical solutions of the fractional system represented in equation (7), and $x_{n}, y_{n}$, and $z_{n}$ are the exact solutions; the residual function with the Caputo derivative is given by the following expression:

$$
\begin{aligned}
& \left|x\left(t_{n}\right)-x_{n}\right|=\mathcal{O}\left(h^{\min \{\alpha+1,2\}}\right), \\
& \left|y\left(t_{n}\right)-y_{n}\right|=\mathcal{O}\left(h^{\min \{\alpha+1,2\}}\right), \\
& \left|z\left(t_{n}\right)-z_{n}\right|=\mathcal{O}\left(h^{\min \{\alpha+1,2\}}\right) .
\end{aligned}
$$

We can notice that the convergence of the numerical scheme presented in this section is obtained when the stepsize $h$ converges to 0 [23]. The stability of our numerical scheme is obtained from the Lipchitz criterion of the functions $u, v$, and $w$.

\section{Illustration of the Numerical Scheme}

This section presents the phase portraits of fractional-order system (7) with commensurate and incommensurate fractional orders. The main objective is to illustrate the numerical scheme presented in previous Section 4 . We set the 
following values for the parameters of the model $a=1$, $b=1$, and $c=1$ and the initial conditions given by equation (9). For Figure 1, the considered order is $\alpha=0.95$.

For more illustrations, we consider system (7) with commensurate order $\alpha=0.90$. The phase portraits are presented in Figure 2.

An interesting procedure of comparison between the geometry of the attractors can be found in [24]. The method consists of comparing the amplitude of the attractors in different orders of the Caputo derivative. The comparison of the attractors' geometry at the orders $\alpha=0.9$ and $\alpha=0.95$ can be analyzed for model (7). We adopt the sketch presented in [24]. We consider the phase portraits in plane $(x, y)$ in Figures 1 and 2 and construct polygons, calculate their areas, and compare them. We have following Figures 3 and 4 .

In Figure 3, amplitudes are $E F=12.2 \mathrm{~cm}$ and $A B=C D=4 \mathrm{~cm}$, and in Figure 4, the amplitudes are $K L=$ $10.88 \mathrm{~cm}$ and $G H=I J=3 \mathrm{~cm}$; referring to the dimension and calculating the areas of the polygons $A F B D E C$ and GLHJKI, we notice that the area $(A F B D E C)>$ area $(G L H J K I)$; thus, there exists a significant difference between the attractors at the orders $\alpha=$ 0.9 and $\alpha=0.95$. The method adopted in this section proves the influence of the order of the Caputo derivative. To see more the impact of the order of the Caputo derivative, we consider fractional chaotic equation (7) with incommensurate order:

$$
\begin{aligned}
x^{\prime} & =a x+y+y z, \\
y^{\prime} & =-x z+y z, \\
D_{c}^{\alpha} z & =-b z-c x y .
\end{aligned}
$$

System (31) is obtained when $\kappa=1$ and $\beta=1$ in equation (7). We have the phase portraits in following Figure 5.

The Caputo derivative's impact can be observed by comparing the phase portrait in the commensurate scenario and the phase portrait in the incommensurate scenario, which have different geometries. To detect equation (7) chaotic behavior with the orders $\alpha=0.9$ and $\alpha=0.95$, we calculate the Lyapunov exponents. The Lyapunov exponents will permit us, in general, to recognize chaotic behaviors. The Jacobian matrix of fractional differential equation (7) with the same order is given by the following matrix, which is a fundamental tool for finding the Lyapunov exponents:

$$
J=\left(\begin{array}{ccc}
a & 1+z & y \\
-z & z & -x+y \\
-c y & -c x & -b
\end{array}\right)
$$

The Lyapunov exponents in the context of fractional calculus in the chaotic system using Matlab code are proposed in Danca's work. Following the same proposed algorithm, the Lyapunov exponents for fractional-order equation (7) with commensurate order when $\alpha=0.95$ are given as follows:

$$
\begin{aligned}
& \mathrm{LYE}_{1}=0.3449, \\
& \mathrm{LYE}_{3}=0, \\
& \mathrm{LYE}_{3}=-2.5326 .
\end{aligned}
$$

And, its associated Kaplan-Yorke dimension is given as follows:

$$
\operatorname{dim}(\mathrm{LYE})=2+\frac{\mathrm{LYE}_{1}+\mathrm{LYE}_{2}}{\left|\mathrm{LYE}_{3}\right|}=2.1362
$$

Following the previous results, we can give the nature of the behaviors. First of all, the fractional system is dissipative because the sum of all Lyapunov exponents gives a negative number. The second property is to detect the chaotic behavior of our presented equation (7) with the same order. We can see with equation (33), we have one positive Lyapunov exponent corresponding to the chaotic behavior of equation (7). We repeat the same analysis with the order $\alpha=0.9$; the Lyapunov exponents are represented as the following form:

$$
\begin{aligned}
& \mathrm{LYE}_{1}=0.3775, \\
& \mathrm{LYE}_{2}=0, \\
& \mathrm{LYE}_{3}=-3.0042 .
\end{aligned}
$$

And, its associated Kaplan-Yorke dimension is given as follows:

$$
\operatorname{dim}(\mathrm{LYE})=2+\frac{\mathrm{LYE}_{1}+\mathrm{LYE}_{2}}{\left|\mathrm{LYE}_{3}\right|}=2.1257
$$

The system is dissipative as well because the addition of all Lyapunov exponents is negative. For the nature of the behaviors, we analyze equation (35); we have the same conclusion as in the previous case, equation (7) has chaotic behavior with the order $\alpha=0.9$. It is caused by the existence of one positive Lyapunov exponent. This section's main conclusion is when the fractional derivative orders vary in the interval $[0.9,1)$, fractional equation (7) with the same order has chaotic behavior. To confirm this assumption, we calculate in following Table 1 the Lyapunov exponents versus the fractional-order derivative's variation into $[0.9,1)$.

Checking the results on Table 1, we conclude that fractional-order equation (7) is dissipative at all orders in the interval $[0.9,1)$. It is because, at all orders, the addition of the Lyapunov exponents is negative. We observe one positive Lyapunov exponent at all orders $\alpha$, which means the fractional-order system's solutions have chaotic behavior.

\section{Stability of the Equilibrium Points in Fractional Context Calculus}

In this part, we discuss the fractional-order system's stability by using the Matignon criterion. It is a famous criterion used in fractional calculus. Stability analysis is one of the fundamental points in the chaotic and hyperchaotic system. In general, all equilibrium points when the system is chaotic fail to be stable. In this section, we consider equation (7) with 
6

Complexity
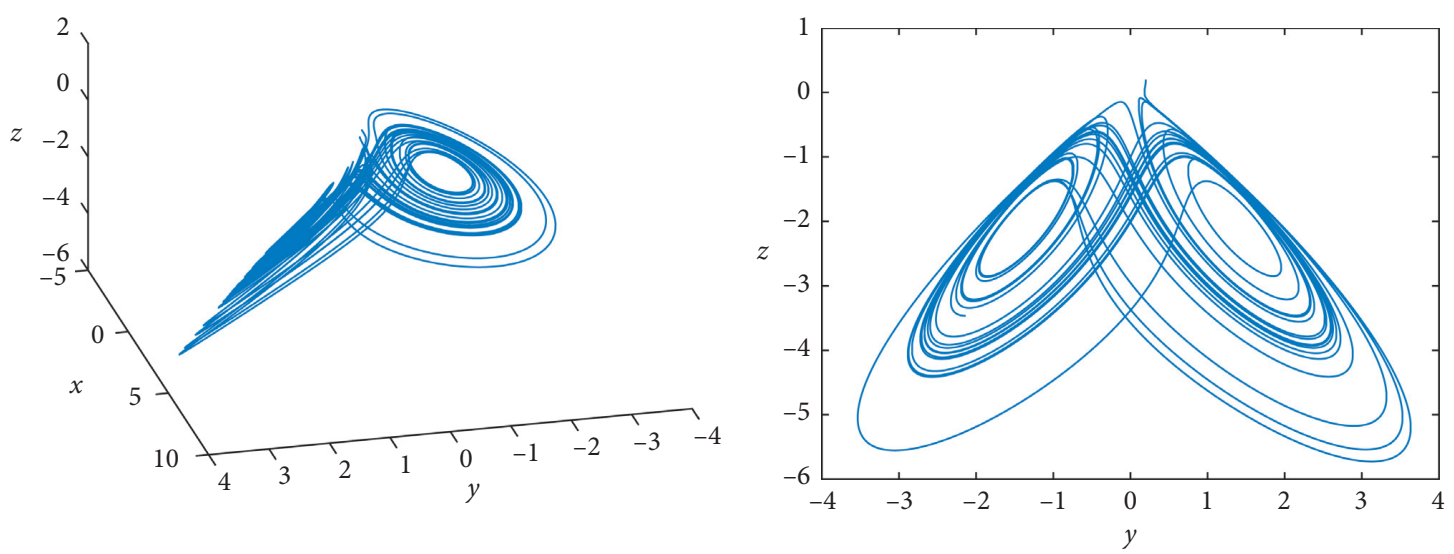

(a)

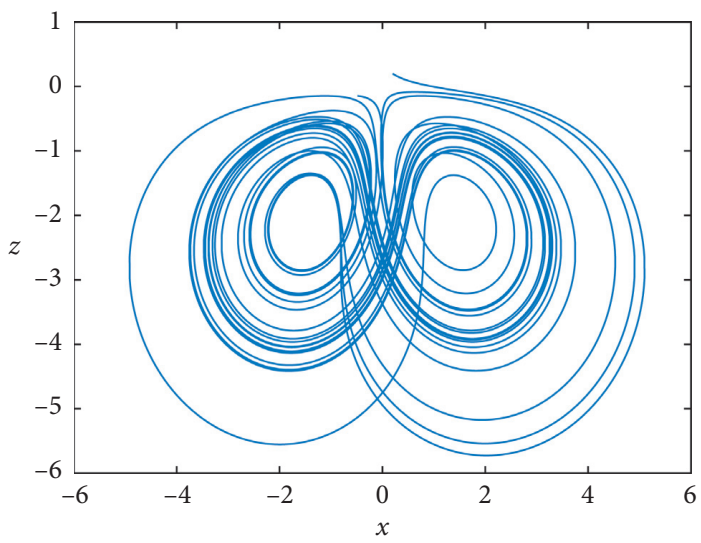

(c) (b)

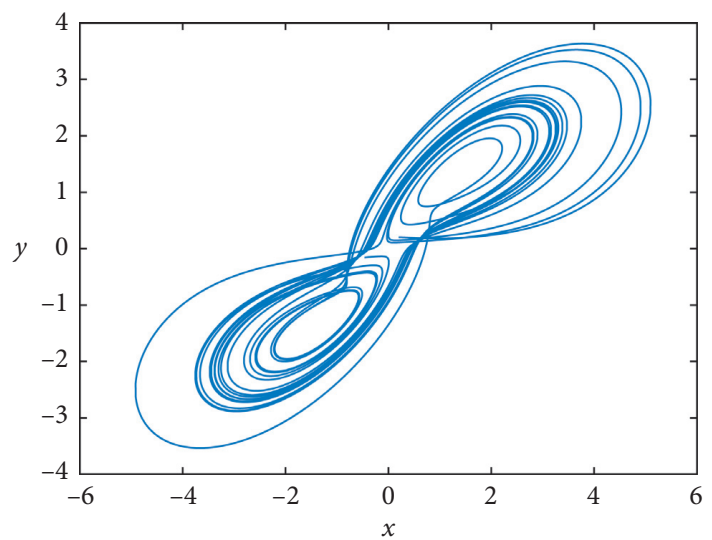

(d)

FIGURE 1: Phase portraits with the order $\alpha=0.95$.
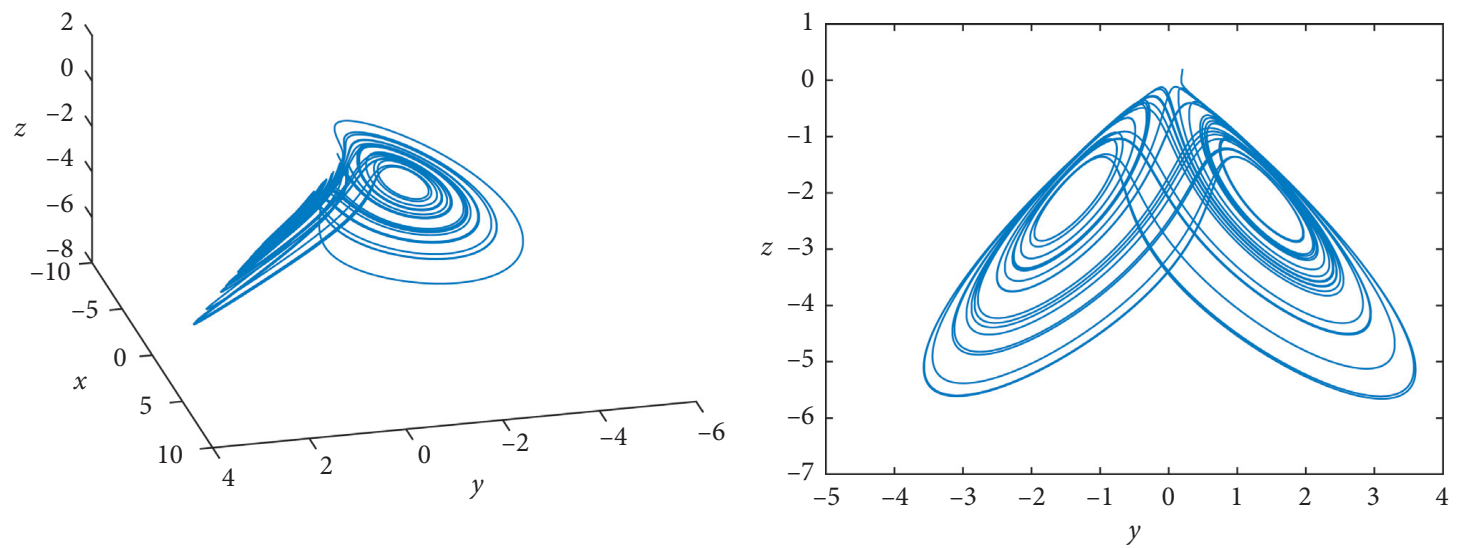

(a)

(b)

FIgURE 2: Continued. 


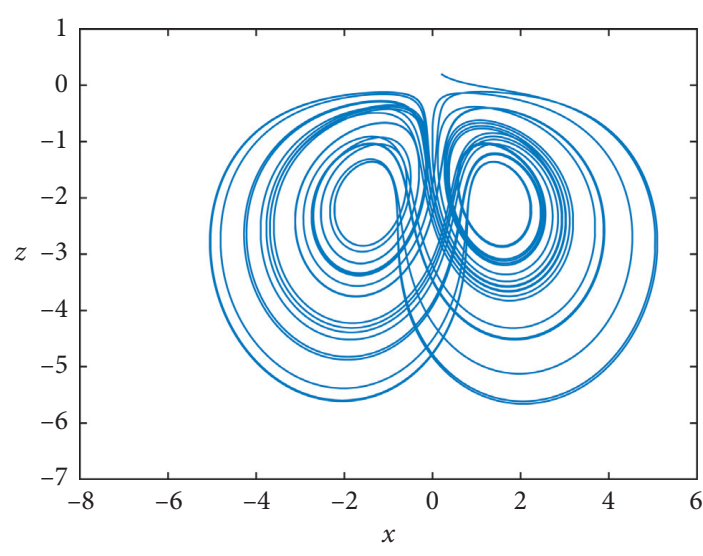

(c)

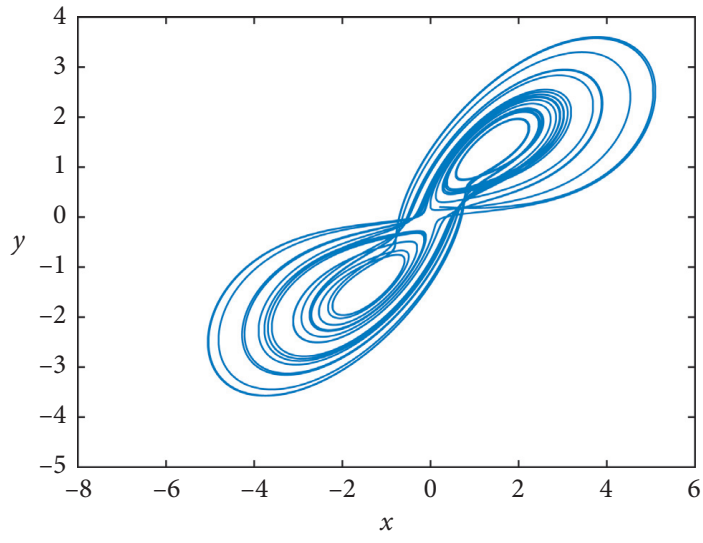

(d)

Figure 2: Phase portraits with the order $\alpha=0.9$.

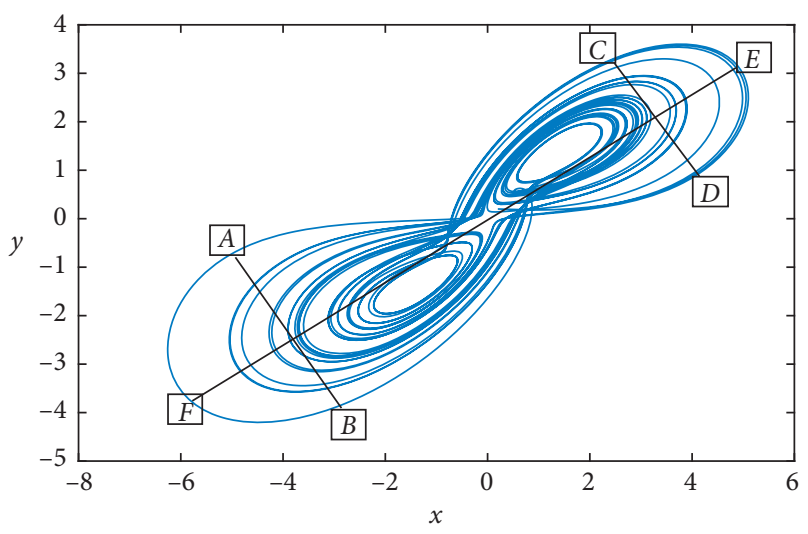

FIgURE 3: Phase spaces with polygon with the order $\alpha=0.9$.

commensurate order. It is also interesting to focus on the stability of its equilibrium points. The Jacobian matrix given in the previous section will be used again. We have the matrix described by the following expression:

$$
J=\left(\begin{array}{ccc}
a & 1+z & y \\
-z & z & -x+y \\
-c y & -c x & -1
\end{array}\right) .
$$

The following points are the real equilibrium points when you exclude the complex eigenvalues:

$$
\begin{aligned}
& P_{1}=(0,0,0), \\
& P_{2}=(-1.41,-1.41,-2), \\
& P_{3}=(1.41,1.41,-2) .
\end{aligned}
$$

The principle of the method consists of evaluating the Jacobian matrix at all points previously mentioned. For the first point $P_{1}$, the Jacobian matrix is as follows:

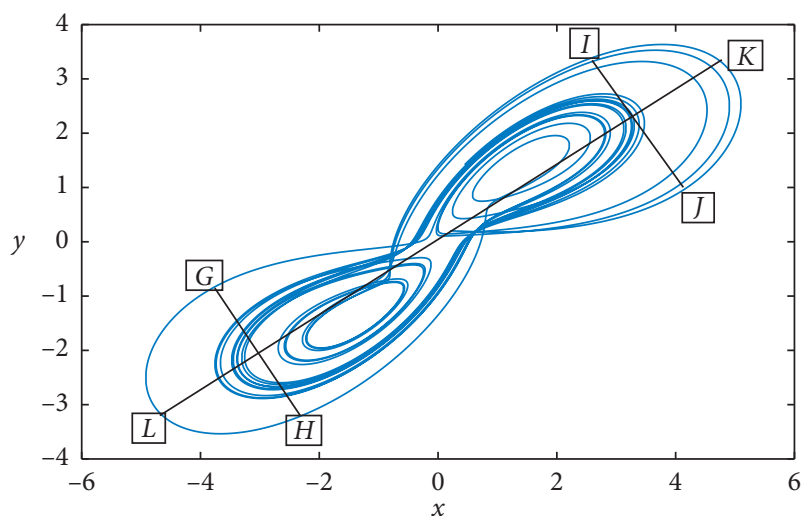

Figure 4: Phase spaces with polygon with the order $\alpha=0.95$.

$$
J\left(P_{1}\right)=\left(\begin{array}{ccc}
1 & 1 & 0 \\
0 & 0 & 0 \\
0 & 0 & -1
\end{array}\right) .
$$

The previous matrix has eigenvalues as the following numbers $\lambda_{1}=1, \lambda_{2}=0$, and $\lambda_{3}=-1$. We notice that the third eigenvalue satisfies $\left|\arg \left(\lambda_{3}\right)\right|=\pi>(\alpha \pi / 2)$. The first eigenvalue obeys that $\left|\arg \left(\lambda_{1}\right)\right| \approx 0<(\alpha \pi / 2)$ for all $\alpha$. Thus, the equilibrium point is not stable for all orders $\alpha$ of the Caputo derivative.

The matrix in equation (37) at the point $P_{2}$ is described as the following form:

$$
J\left(P_{2}\right)=\left(\begin{array}{ccc}
1 & -1 & -1.41 \\
2 & -2 & 0 \\
1.41 & 1.41 & -1
\end{array}\right) .
$$

The previous matrix has eigenvalues as the following numbers $\lambda_{1}=0.1230+1.8776 i, \lambda_{2}=0.1230-1.8776 i$, and $\lambda_{3}=-2.246$. We remark that the third eigenvalue satisfies $\left|\arg \left(\lambda_{3}\right)\right|=\pi>(\alpha \pi / 2)$. The first eigenvalue obeys to 


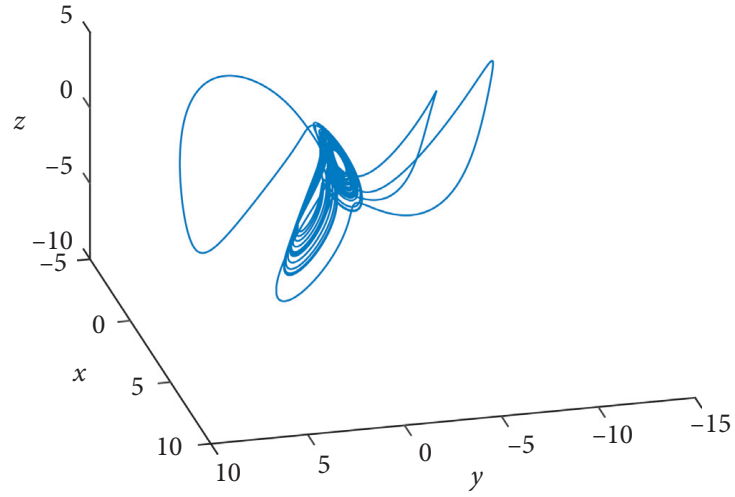

(a)

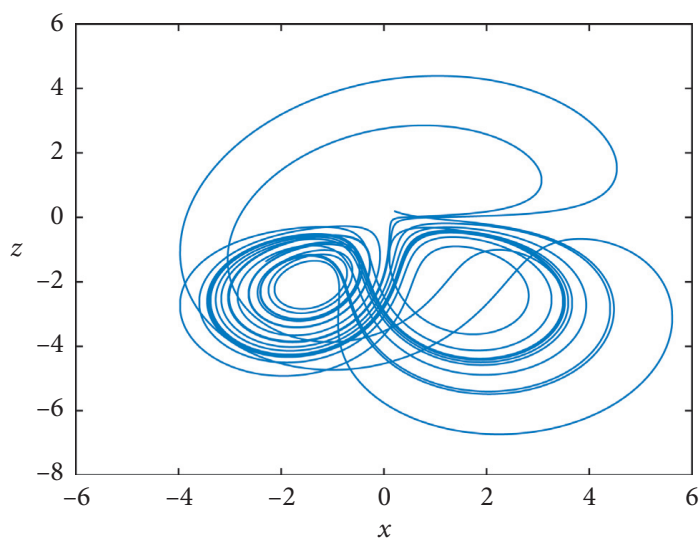

(c)

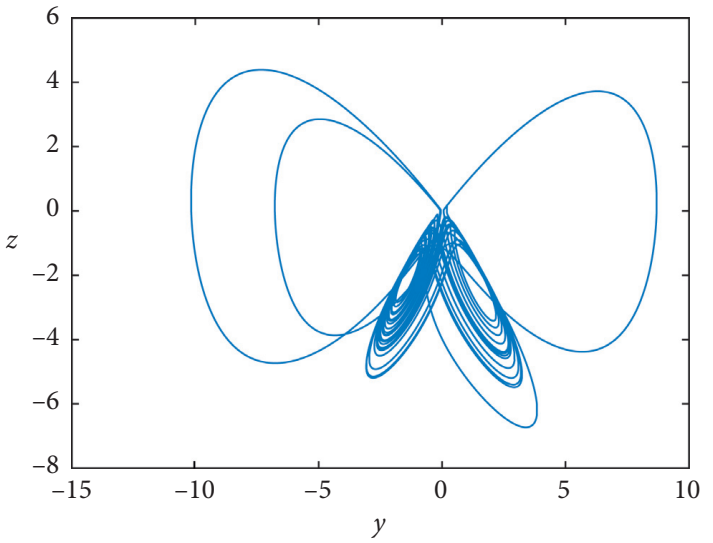

(b)

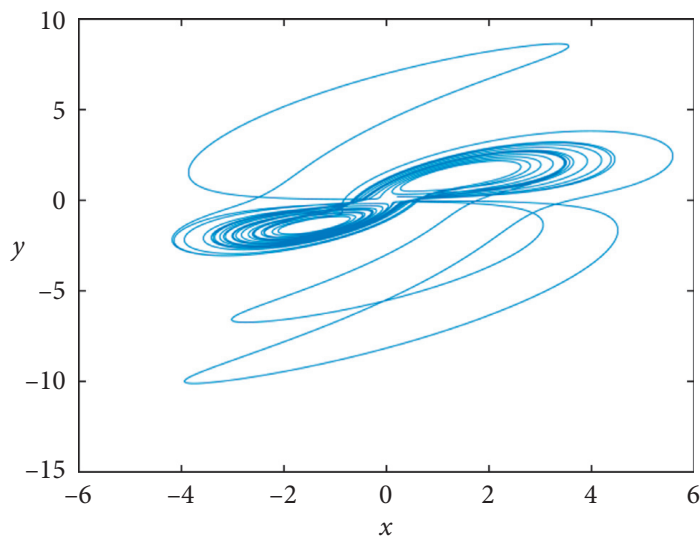

(d)

FIgure 5: Phase portraits with the order $\alpha=0.9$ in equations (32)-(34).

TABLE 1: Lyapunov exponents.

\begin{tabular}{lccc}
\hline$\alpha$ & $\mathrm{LE}_{1}$ & $\mathrm{LE}_{2}$ & $\mathrm{LE}_{3}$ \\
\hline 0.90 & 0.3775 & 0 & -3.0042 \\
0.91 & 0.3581 & 0 & -2.7858 \\
0.92 & 0.3083 & 0 & -2.8379 \\
0.93 & 0.2701 & 0 & -2.7337 \\
0.94 & 0.2979 & 0 & -2.5546 \\
0.95 & 0.3449 & 0 & -2.5326 \\
0.96 & 0.2177 & 0 & -2.3049 \\
0.97 & 0.2107 & 0 & -2.1671 \\
0.98 & 0.2171 & 0 & -2.0931 \\
0.99 & 0.2402 & 0 & -2.0701 \\
0.9950 & 0.2215 & 0 & -1.9927 \\
\hline
\end{tabular}

$\left|\arg \left(\lambda_{1}\right)\right| \approx(43 \pi / 90)>(\alpha \pi / 2)$ for all $\alpha<0.9$ and the last eigenvalue $\left|\arg \left(\lambda_{2}\right)\right| \approx(43 \pi / 90)>(\alpha \pi / 2)$ for all $\alpha$. Thus, the equilibrium point is not stable when $\alpha$ exceeds 0.9 .

The Jacobian at the point $P_{3}$ is described by

$$
J\left(P_{2}\right)=\left(\begin{array}{ccc}
1 & -1 & 1.41 \\
2 & -2 & 0 \\
-1.41 & -1.41 & -1
\end{array}\right) \text {. }
$$

The previous matrix has eigenvalues as the following numbers $\lambda_{1}=0.1230+1.8776 i, \lambda_{2}=0.1230-1.8776 i$, and $\lambda_{3}=-2.246$. We have that the third eigenvalue satisfies $\left|\arg \left(\lambda_{3}\right)\right|=\pi>(\alpha \pi / 2)$. The first eigenvalue obeys that $\left|\arg \left(\lambda_{1}\right)\right| \approx(43 \pi / 90)>(\alpha \pi / 2)$ for all $\alpha<0.9$ and the last $\left|\arg \left(\lambda_{2}\right)\right| \approx(43 \pi / 90)>(\alpha \pi / 2)$ for all $\alpha$. Then, the equilibrium point is not stable when $\alpha$ exceeds 0.9 .

\section{Bifurcation Diagrams and Lyapunov Exponents}

This section analyzes the impact caused by the variation of the parameters $a$ and $c$ on chaotic behavior. Small variations are applied to $a$ and $c$, and we depict the responses via the bifurcation diagrams plus phase portraits. In this section, for more clarity in the curve, we consider the step-size $h=0.005$. All findings are obtained with equation (7) in commensurate order.

We begin our analysis with the variation of the parameter $a$ in the interval $(0,5)$. We fix $b=1$ and $c=1$. In Figure 6 , we represent the bifurcation diagram versus the parameter $a$.

We notice that when the parameter $a$ varies in the region $[0.6,5)$, fractional system $(5)-(8)$ has chaotic behavior. Note that the variation of the parameter $a$ into 
$(0,0.5)$ generates the solutions' convergence to a stable equilibrium point. For an illustration of the chaotic behavior, we represent the phase portraits of our system at $a=1.5$ and fixing $b=1$ and $c=1$ at the order $\alpha=0.95$, see Figure 7.

We confirm the chaotic behavior with the existence of one positive and large Lyapunov exponent:

$$
\begin{aligned}
& \mathrm{LYE}_{1}=0.1918, \\
& \mathrm{LYE}_{2}=0, \\
& \mathrm{LYE}_{3}=-2.4806 .
\end{aligned}
$$
follows:

And, its associated Kaplan-Yorke dimension is given as

$$
\operatorname{dim}(\mathrm{LYE})=2+\frac{\mathrm{LYE}_{1}+\mathrm{LYE}_{2}}{\left|\mathrm{LYE}_{3}\right|}=2.0773
$$

To validate the convergence of the solutions to a stable equilibrium point, we study the local stability of the nontrivial equilibrium point of equation (7) with commensurate order considering $a=0.4$. The equilibrium points of equation (7) with commensurate order with $a=0.4$ are given by $O(0,0,0), \quad A(-1.18,-1.18,-1.4)$, and $B(1.18,1.18,-1.4)$. The Jacobian matrix at the point $\mathrm{O}(0,0,0)$ gives

$$
J(O)=\left(\begin{array}{ccc}
0.4 & 1 & 0 \\
0 & 0 & 0 \\
0 & 0 & -1
\end{array}\right) .
$$

The eigenvalues of the previous matrix are given by $\lambda_{1}=$ $0.4, \lambda_{2}=0$, and $\lambda_{3}=-1$. We can remark that the first eigenvalue does not obey to Matignon criterion; thus, the point $O(0,0,0)$ is not local stable. Thus, the convergence of the solutions of equation (7) is not at the point $O(0,0,0)$. We now try to a point $A(-1.18,-1.18,-1.4)$; thus, the Jacobian matrix is given by

$$
J(A)=\left(\begin{array}{ccc}
0.4 & -0.4 & -1.18 \\
1.4 & -1.4 & 0 \\
1.18 & 1.18 & -1
\end{array}\right) .
$$

We obtain as eigenvalues the following values $\lambda_{1}=-0.0763+1.4507 i, \quad \lambda_{2}=-0.0763-1.4507 i, \quad$ and $\lambda_{3}=-1.8474$. We can observe that all eigenvalues have negative real parts. Thus, the Matignon criterion is satisfied by all eigenvalues. Thus, the point $A(-1.18,-1.18,-1.4)$ is a stable equilibrium point. We finish with the point $B(1.18,1.18,-1.4)$. The Jacobian matrix at the point $B$ is described as the following form:

$$
J(C)=\left(\begin{array}{ccc}
0.4 & -0.4 & 1.18 \\
1.4 & -1.4 & 0 \\
-1.18 & -1.18 & -1
\end{array}\right) \text {. }
$$

The eigenvalues of the previous Jacobian matrix at this point are given by $\lambda_{1}=-0.0763+1.4507 i$, $\lambda_{2}=-0.0763-1.4507 i$, and $\lambda_{3}=-1.8474$. We can observe that all eigenvalues have negative real parts; thus, the Matignon criterion is automatically satisfied. Thus, the point $C$ coincides with the stable equilibrium point. Referring to the phase portraits, the trajectories converge to the point $B$ when $a=0.4$.

We finish this section by the bifurcation diagram generated by the variation of the parameter $c \in(0,25)$, and we illustrate the impact of the parameter $c$ on the phase portraits, see Figure 8, taken at $a=1$ and $c=4$.

The bifurcation diagram shown in Figure 9 indicates that , in our considered interval $(0,25)$, the system has continuously chaotic behavior. Let us $c=4$; the chaotic behavior at this point is confirmed by the existence of one positive Lyapunov exponent; we have the following calculations:

$$
\begin{aligned}
& \mathrm{LYE}_{1}=0.2485, \\
& \mathrm{LYE}_{2}=0, \\
& \mathrm{LYE}_{3}=-2.4311 .
\end{aligned}
$$

And, its associated Kaplan-Yorke dimension is given as follows:

$$
\operatorname{dim}(\mathrm{LYE})=2+\frac{\mathrm{LYE}_{1}+\mathrm{LYE}_{2}}{\left|\mathrm{LYE}_{3}\right|}=2.1022
$$

For more confirmation of the chaotic region, we depict our fractional system's phase portraits at $a=1$ and $c=0.5$, see Figure 10.

\section{Electrical Circuit Schematic of the Fractional System}

In this section, we give the circuit representation associated to chaotic system (7) with incommensurate order. For more details in the context of the fractional chaotic circuit, we consider equation (7) with $\kappa=\beta=1$ written using electronic tools:

$$
\begin{aligned}
x^{\prime} & =\frac{1}{R_{1} C_{1}} x+\frac{1}{R_{2} C_{1}} y+\frac{1}{R_{3} C_{1}} y z, \\
y^{\prime} & =-\frac{1}{R_{4} C_{1}} x z+\frac{1}{R_{5} C_{2}} y z, \\
D_{c}^{\alpha} z & =-\frac{1}{R_{6} C_{3}} z-\frac{1}{R_{7} C_{3}} x y,
\end{aligned}
$$

where $C_{i}$ for $i=1, \ldots, n$ denotes the value of the capacitors and $R_{i}$ represents the resistance value. To connect the results in this paper with the experimental results, we draw the circuit schematic and generate the phase portraits using oscilloscopes. We first use our numerical scheme in the previous sections to draw the phase portraits. We consider the order of the Caputo derivative as $\alpha=0.95$. The portraits of model (49)-(51) are represented in following Figure 11.

For the circuit schematic, the values of the capacitors are given by $C_{1}=C_{2}=C_{3}=1 \mathrm{nF}$, and the values of the resistors are given $R_{1}=250 \mathrm{k} \Omega, R_{2}=250 \mathrm{k} \Omega, R_{3}=6.25 \mathrm{k} \Omega$, $R_{4}=6.25 \mathrm{k} \Omega, R_{5}=6.25 \mathrm{k} \Omega, R_{6}=250 \mathrm{k} \Omega$, and $R_{7}=6.25 \mathrm{k} \Omega$. 


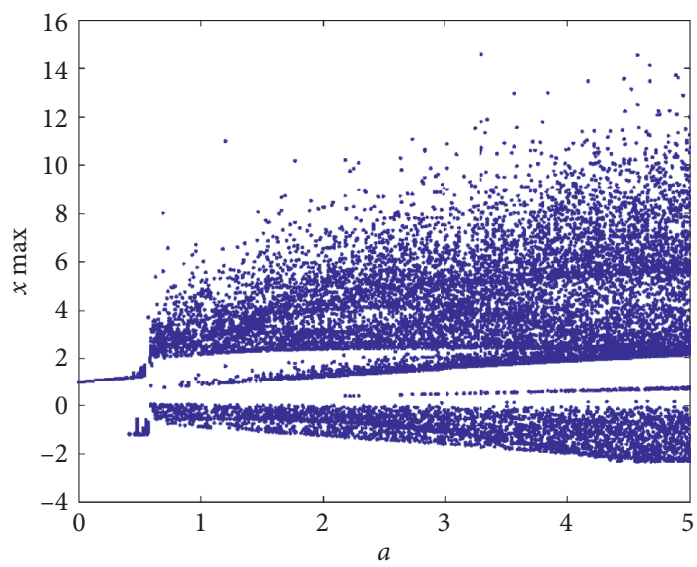

FiguRE 6: Bifurcation diagram versus the parameter $a$ at order $\alpha=0.95$.

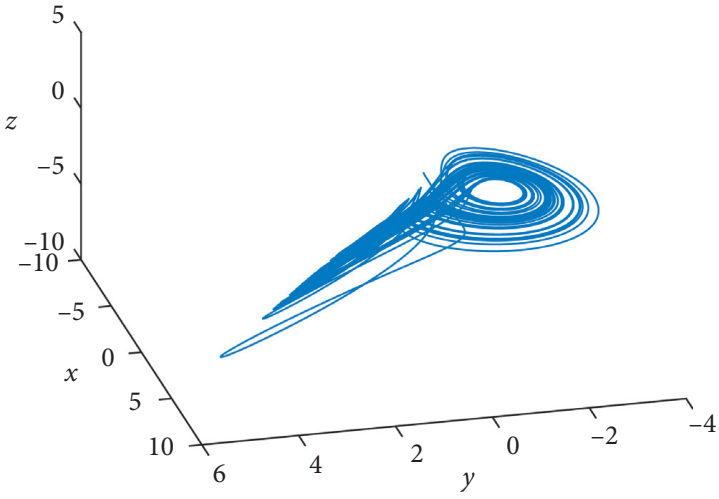

(a)

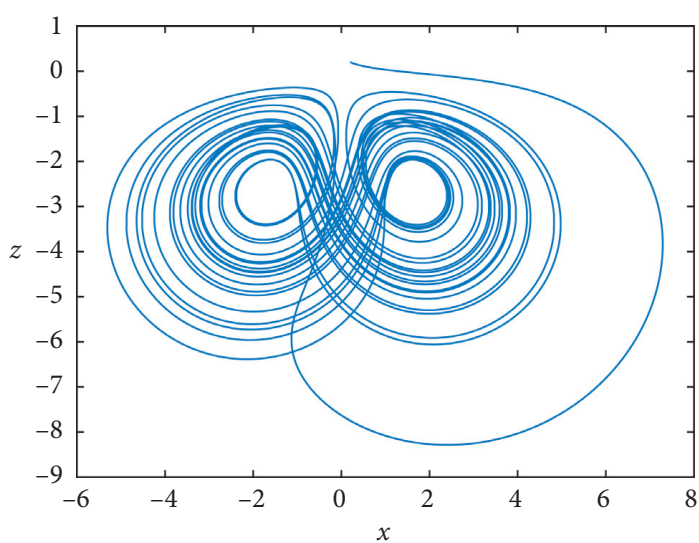

(c)

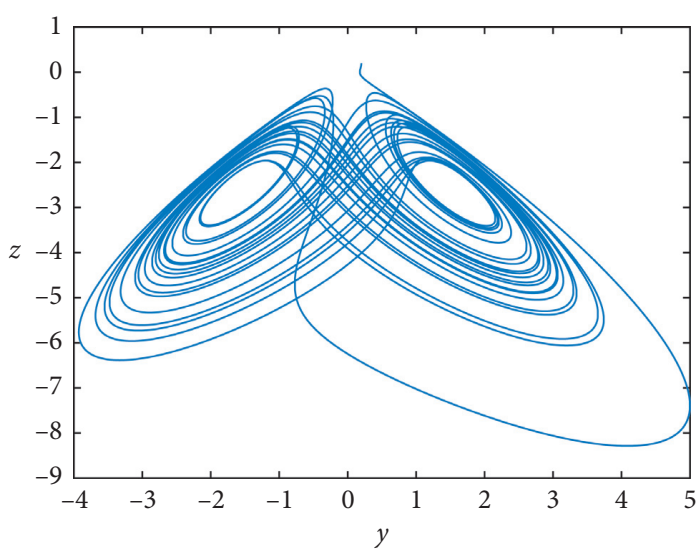

(b)

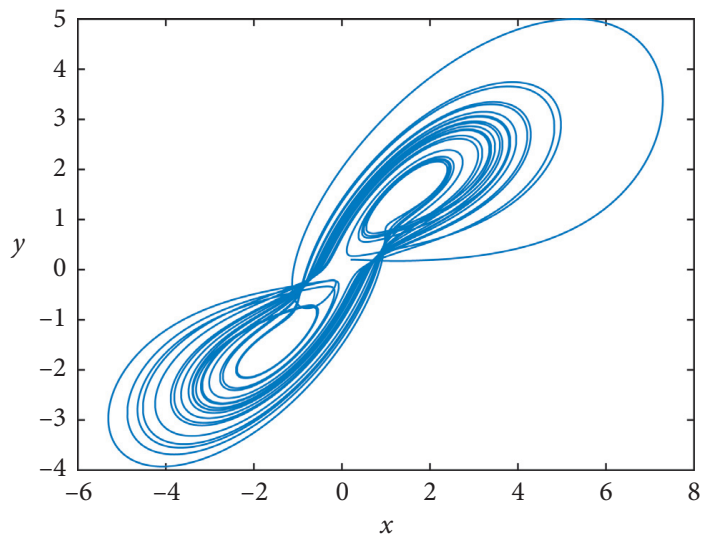

(d)

FIgURE 7: Phase portraits with the order $\alpha=0.95$ and $a=1.5$.

We need to utilize the fractional integrators associated with the order $\alpha=0.95$ [25]; we have the following formula:

$$
\frac{1}{s^{0.95}} \approx \frac{1.2862 s^{2}+18.6004 s+2.0833}{s^{3}+18.4738 s^{2}+2.6547 s+0.003} .
$$

In equation (52), the third-order approximation is used, but more suitable approximations can also be used. This section's objective is the circuit schematic to validate our theoretical results; therefore, third- or fourth-order approximations can be used for illustration. The method of 


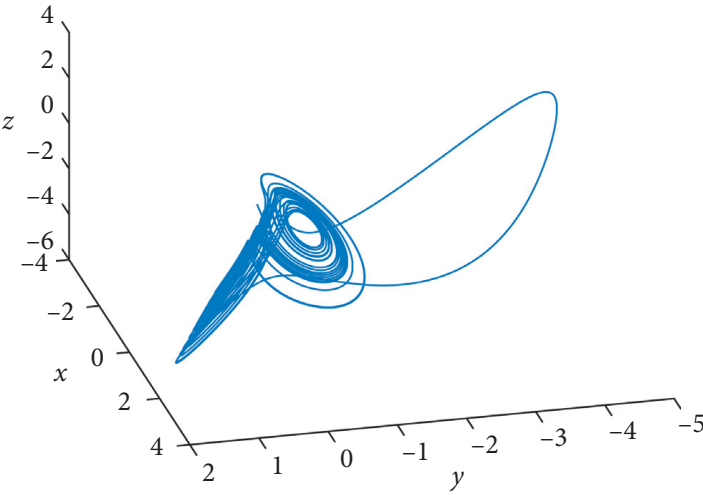

(a)

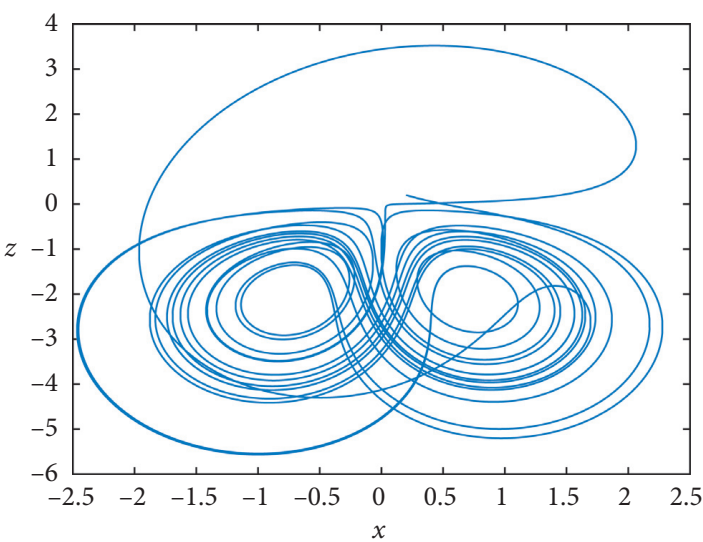

(c)

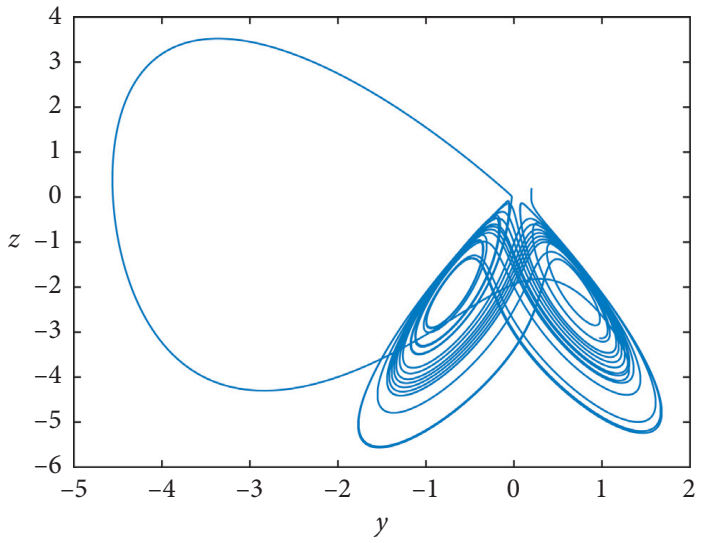

(b)

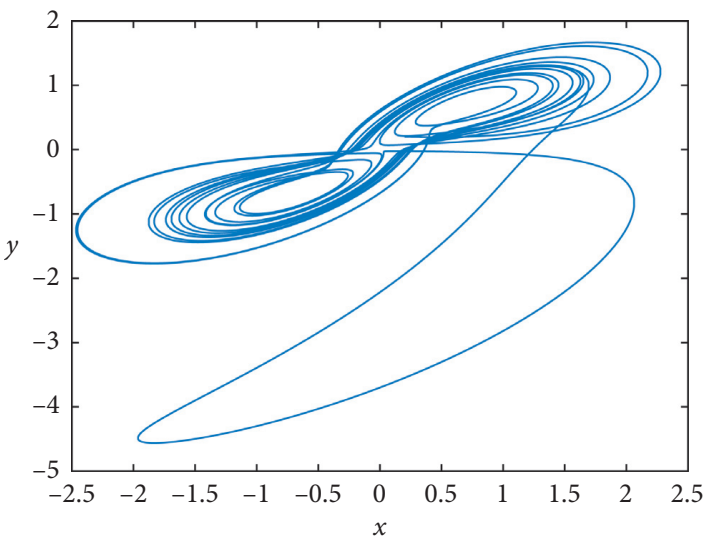

(d)

FIGURE 8: Phase portraits with the order $\alpha=0.95$.

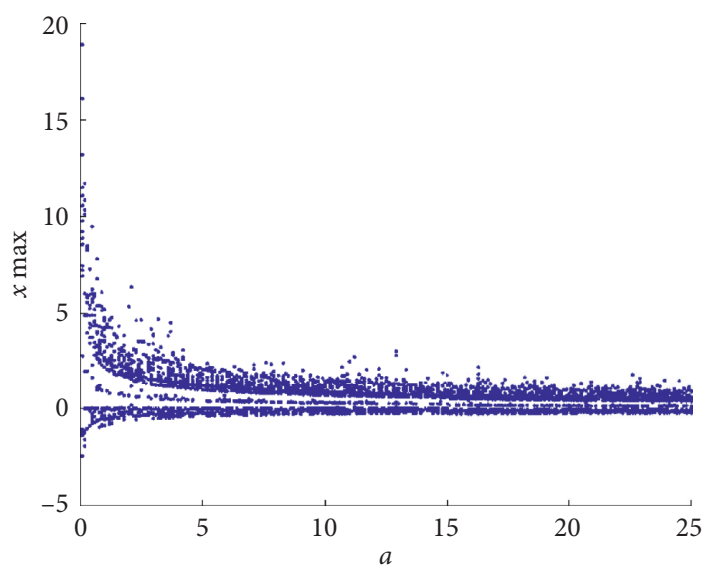

FIgURE 9: Bifurcation diagram versus the parameter $c$ at order $\kappa=0.95$. 


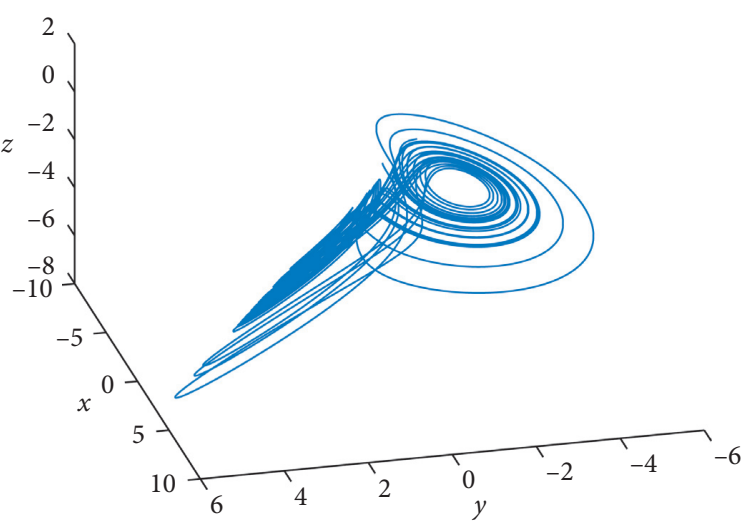

(a)

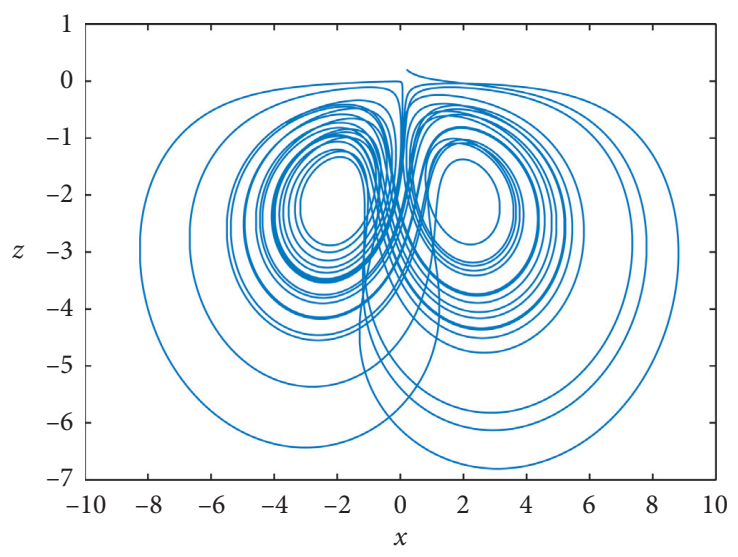

(c)

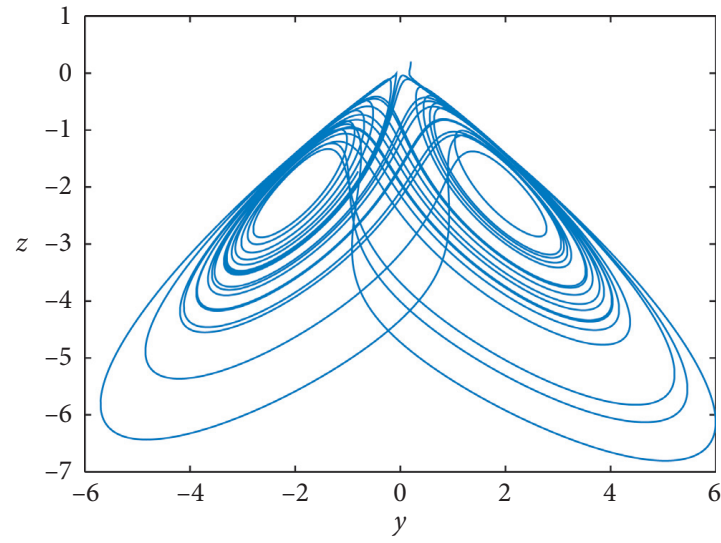

(b)

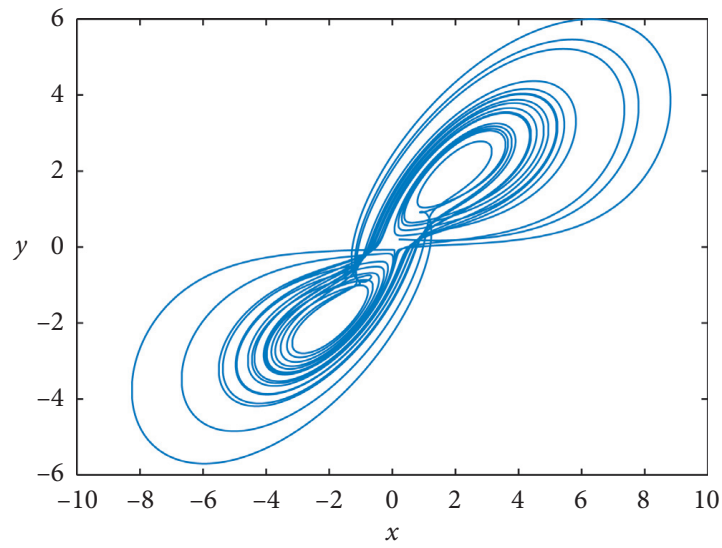

(d)

Figure 10: Phase portraits with the order $\alpha=0.95$.

approximation in equation (52) is not unique. There also exists the $4^{\text {th }}$-order approximation, which can generate a satisfactory magnitude and phase response over a considerable frequency band [26]. For more related works addressing the approximation of the fractional-order capacitor via fourth-order continued fraction expansion, see Mishra et al.'s investigation [26]. Utilizing the transfer function, the above function can be written as the following formula:

$$
T(s)=\frac{\left(1 / C_{3}\right)}{s+\left(1 / R_{9} C_{3}\right)}+\frac{\left(1 / C_{4}\right)}{s+\left(1 / R_{10} C_{4}\right)}+\frac{\left(1 / C_{5}\right)}{s+\left(1 / R_{15} C_{5}\right)} .
$$

Identifying two above equations (45) and (46), we have the following values for the resistors and the capacitors for the fractional integrator, $C_{3}=1.27 \mathrm{nF}, C_{4}=4.7 \mathrm{nF}$, $C_{5}=3.63 \mathrm{nF}, R_{9}=700 \mathrm{M} \Omega, R_{10}=1.5 \mathrm{M} \Omega$, and $R_{15}=15 \mathrm{k} \Omega$. The circuit schematic is done with the aids of 14 resistors, five capacitors, four multipliers for the 4 nonlinear functions, and others, see Figure 12.
The results corresponding to the simulation of the electronic circuit in the Multisim platform are shown in following Figures 13-15.

We can observe that the results with Matlab after simulating the numerical scheme in Figure 11 and the findings corresponding to the simulation of the electronic circuit in the Multisim platform shown in Figures 13-15 are in good agreement. This section connects the theoretical results with experimental results.

Before closing this section, we will implement the integer version of model equations (49)-(51). Therefore, the order $\alpha$ should be $\alpha=1$. For the confirmation of our results obtained after the simulation of the electronic circuit in the Multisim platform, we first simulate the following differential equations:

$$
\begin{aligned}
& x^{\prime}=a x+y+y z, \\
& y^{\prime}=-x z+y z,
\end{aligned}
$$



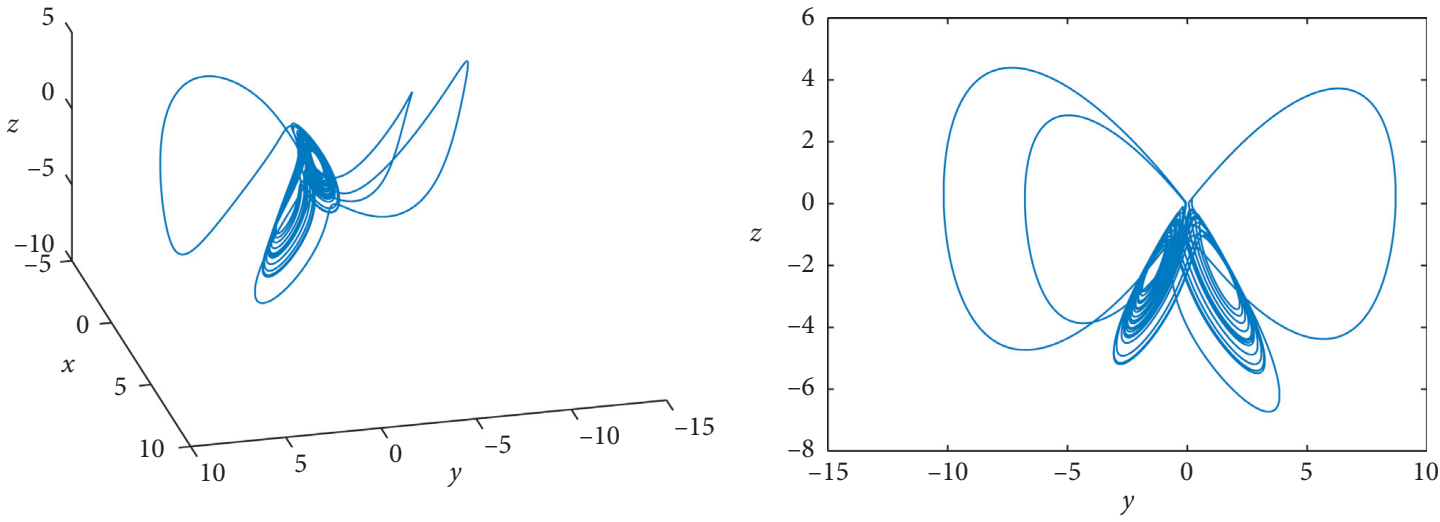

(a)

(b)

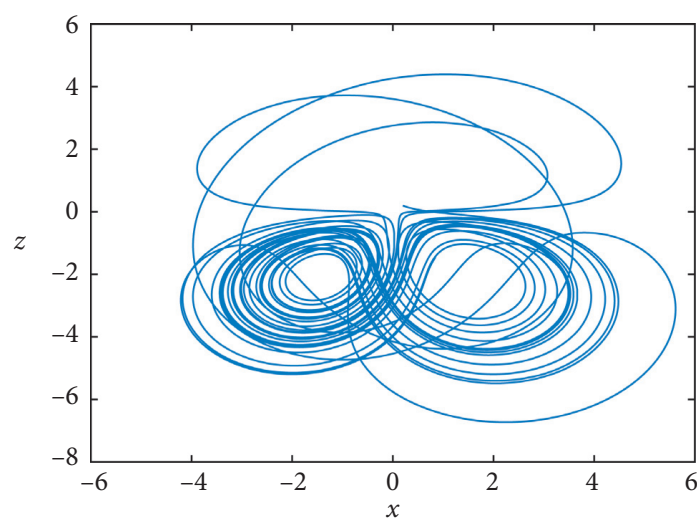

(c)

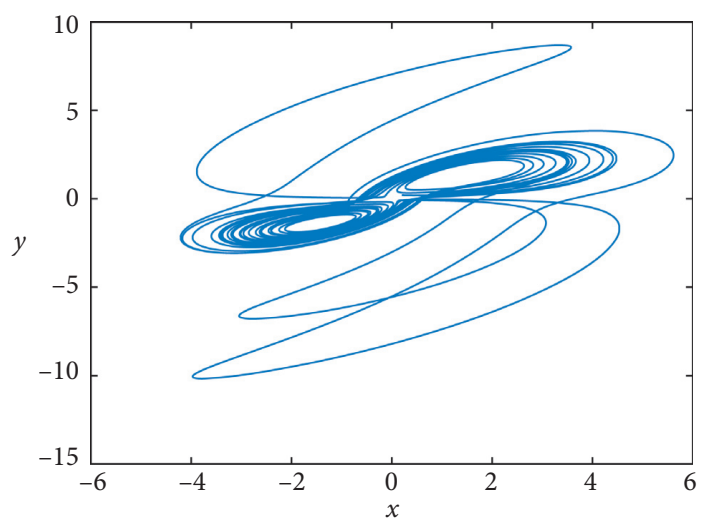

(d)

FIGURE 11: Phase portraits with incommensurate fractional system (42)-(44).

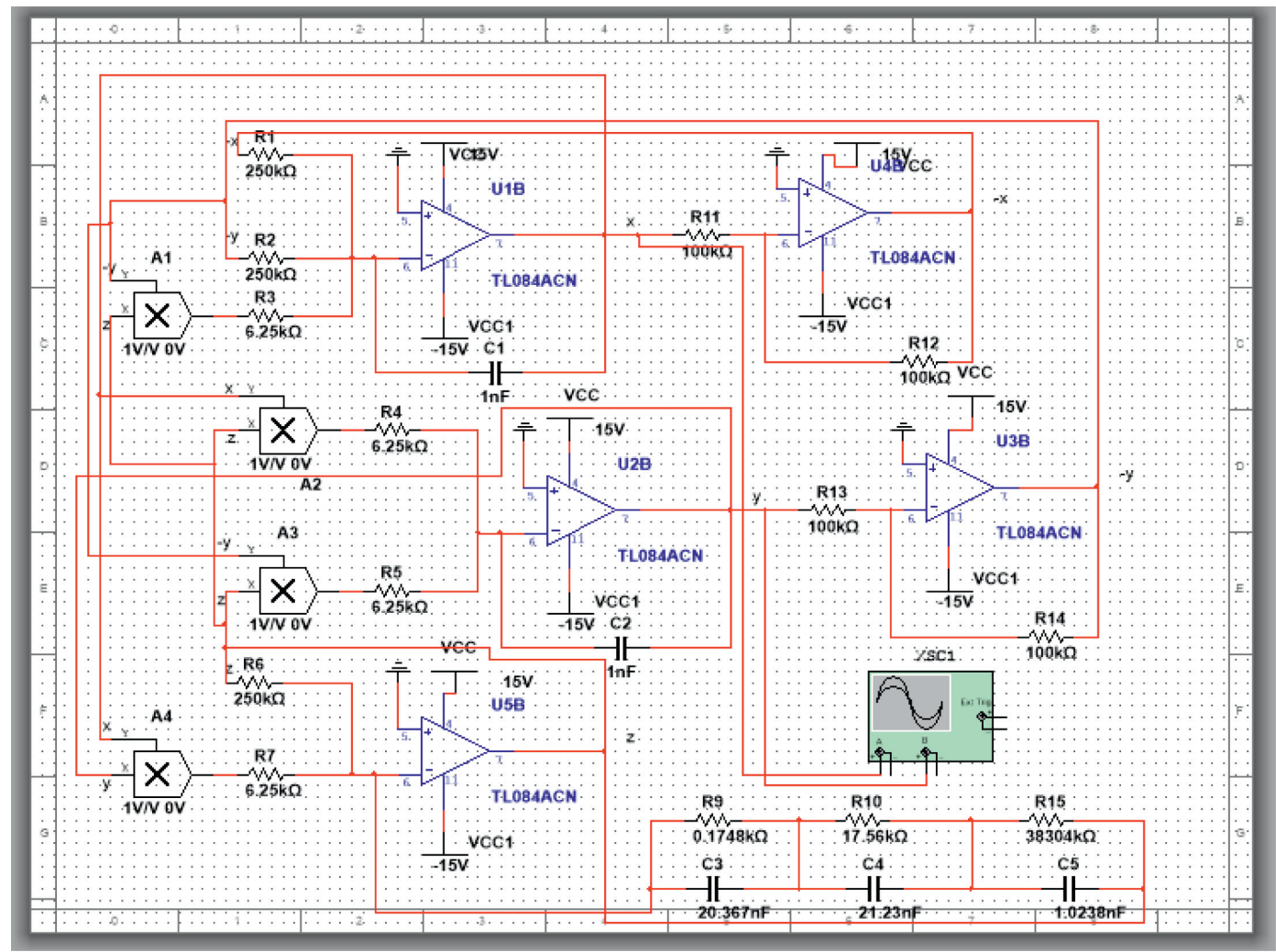

Figure 12: Circuit schematic for fractional-order system (42)-(44). 


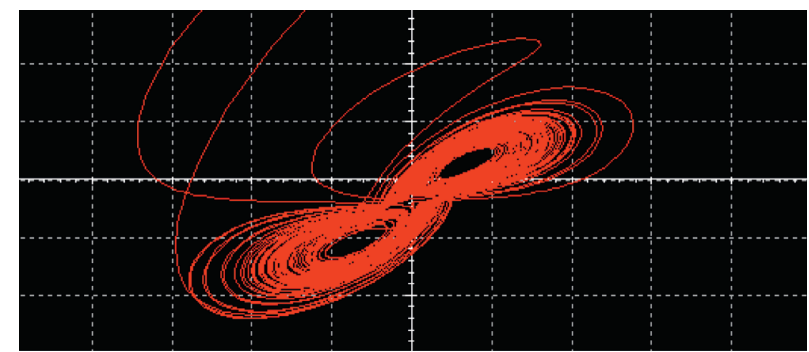

FIgURE 13: $(x, y)$ space portrait of the Multisim simulation of equations (49)-(51).

$$
z^{\prime}=-z-c x y
$$

Under the initial conditions given by system (9) and with $a=1$ and $c=1$, the phase portraits are represented in following Figure 16.

For simulation of system (54)-(56), we rewrite equations (54)-(56) by using resistors and capacitors; we have the following representation:

$$
\begin{aligned}
& x^{\prime}=\frac{1}{R_{1} C_{1}} x+\frac{1}{R_{2} C_{1}} y+\frac{1}{R_{3} C_{1}} y z, \\
& y^{\prime}=-\frac{1}{R_{4} C_{1}} x z+\frac{1}{R_{5} C_{2}} y z, \\
& z^{\prime}=-\frac{1}{R_{6} C_{3}} z-\frac{1}{R_{7} C_{3}} x y,
\end{aligned}
$$

where the values of capacitors are given $C_{1}=C_{2}=C_{3}=1 \mathrm{nF}$ and resistors are given by $R_{1}=250 \mathrm{k} \Omega, R_{2}=250 \mathrm{k} \Omega$, $R_{3}=6.25 \mathrm{k} \Omega, R_{4}=6.25 \mathrm{k} \Omega, R_{5}=6.25 \mathrm{k} \Omega, R_{6}=250 \mathrm{k} \Omega$, and $R_{7}=6.25 \mathrm{k} \Omega$. The schematic of the above system is represented in following Figure 17.

The phase portraits obtained after the simulation of the circuit in Figure 17 are in following Figures 18-20.

We can observe that the theoretical results 16 and the experimental results 18, 19, and 20 are in good agreement.

\section{Sensitivity to Initial Conditions}

In this section, we study the sensitivity of our model when the initial conditions change permanently. By the definition of chaos, the chaotic systems are very sensitive to the starting conditions. In this section, we try to measure the impact of the initial conditions via the bifurcation diagrams. For the rest of the paper, we consider equation (7) with commensurate order $\alpha=0.95$. The comparisons are made with the conditions given by

$$
\begin{aligned}
& x(0)=x_{0}=0.2, \\
& y(0)=y_{0}=0.2, \\
& z(0)=z_{0}=0.2 .
\end{aligned}
$$

We first consider the variation of the parameter $a$, and we influence the last initial condition $z_{0}$. The bifurcation map21 associated with the initial conditions $(0.2,0.2,0.2)$ (blue) and $(0.2,0.2,0.5)$ (red) versus the variation of the parameter $a$ is given in following Figure 21 .

The present bifurcation diagram, see Figure 21, informs us that the chaotic regions do not change significantly when the parameter $a$ varies. Note that, for the initial condition $(0.2,0.2,0.2)$, the chaotic region is $(0.6,5)$, and for the initial condition $(0.2,0.2,0.5)$, the chaotic region is $(0.7,5)$. The initial conditions' impact can be observed at the beginning of the bifurcation diagram, in the interval $(0,1)$, where system (7) trajectories converge to a stable equilibrium point. We notice that, with the first initial condition $(0.2,0.2,0.2)$, the system enters first in the chaotic region. The small perturbation on the starting condition can generate changes but not significantly when the parameter varies. To see the initial condition's influence according to the variation of $a$, we illustrate by representing the phase portraits at $a=0.6$ and $a=0.7$. When $a=0.6$, we can observe that, in Figure 22, the phase space in $(x, y)$ plane (blue) corresponding to $(0.2,0.2,0.2)$ is chaotic, contrary to the phase space 22 in plane $(x, y)$ (red) corresponding to $(0.2,0.2,0.5)$, where clearly the solutions of the system converge to an stable equilibrium point. Let us now $a=0.7$; in Figure 22, the phase space in the plane $(x, y)$ (blue) corresponding to the condition $(0.2,0.2,0.2)$ is chaotic, and the phase space 22 in the plane $(x, y)$ (red) corresponding to $(0.2,0.2,0.5)$ is also chaotic.

In the second, we suppose $y_{0}=0.5, x_{0}=0.2$, and $z_{0}=$ 0.2 are fixed. The variation of the parameter $a$ is also considered in the bifurcation diagram. The sensitivity to the starting condition can also be observed in the bifurcation diagram. The bifurcation diagram, see Figure 23, associated with the conditions $(0.2,0.2,0.2)$ (blue) and $(0.2,0.2,0.5)$ (red) versus the variation of the parameter $a$ is given in following Figure 23.

The bifurcation diagram, see Figure 23, informs us the chaotic regions $(0.6,5)$ for the starting condition of equation (7) are given by $(0.2,0.2,0.2)$ and $(0.7,5)$ for the new condition $(0.2,0.5,0.2)$. The influence of the initial conditions can be noticed in the interval $(0.6,0.7)$, after this region equation (7) has strange attractors with the conditions $(0.2,0.2,0.2)$ and $(0.2,0.5,0.2)$. For illustration, see Figure 24 , we consider $a=0.65$, and we represent the phase space in the plane $(x, z)$ for more clarity.

We finish this section with the perturbation of the first initial condition. The bifurcation diagram, see Figure 25, 


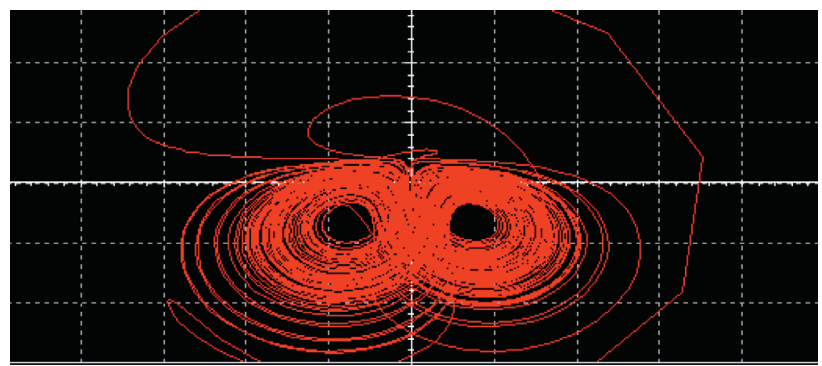

Figure 14: $(x, z)$ space portrait of the Multisim simulation of equations (49)-(51).

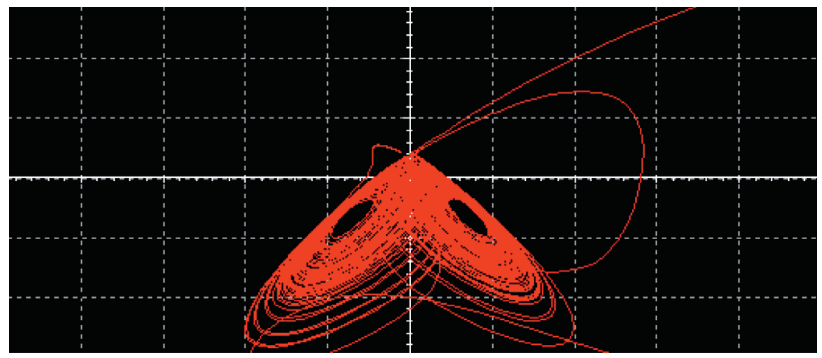

Figure 15: $(y, z)$ space portrait of the Multisim simulation of equations (49)-(51).

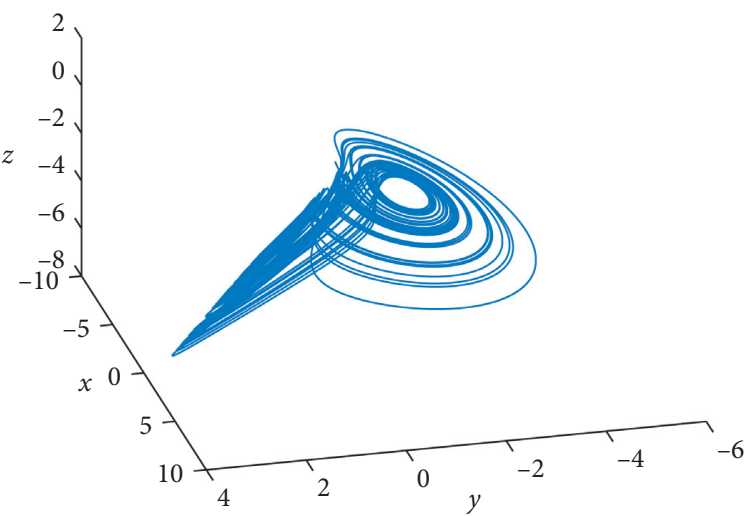

(a)

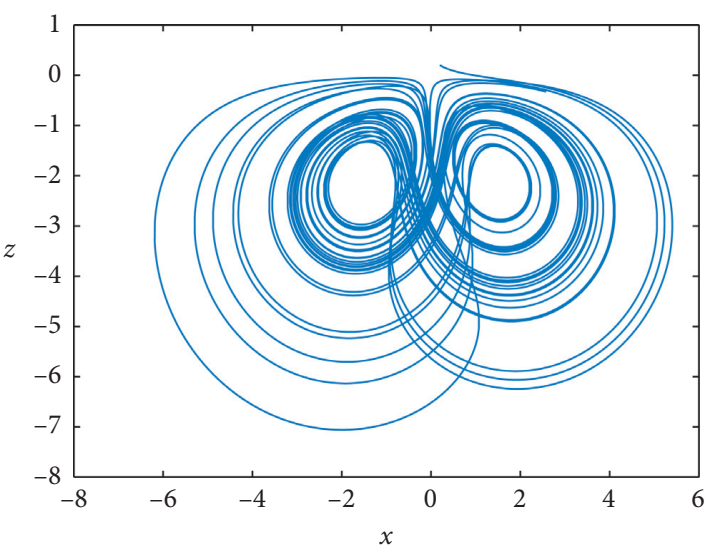

(c)

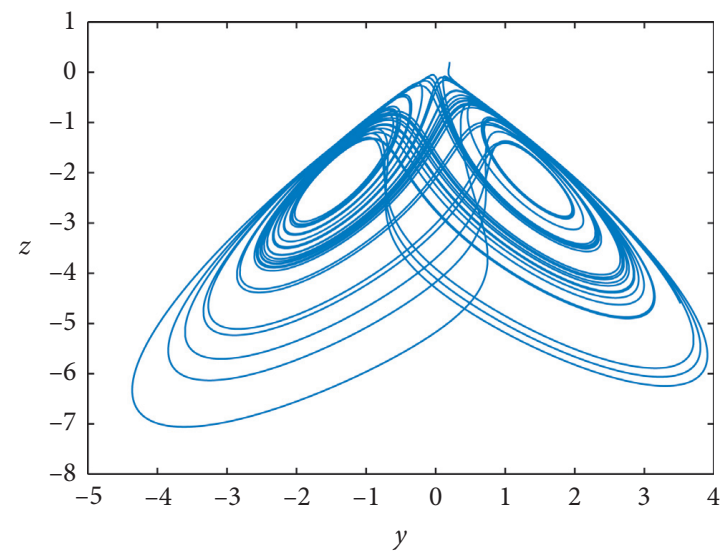

(b)

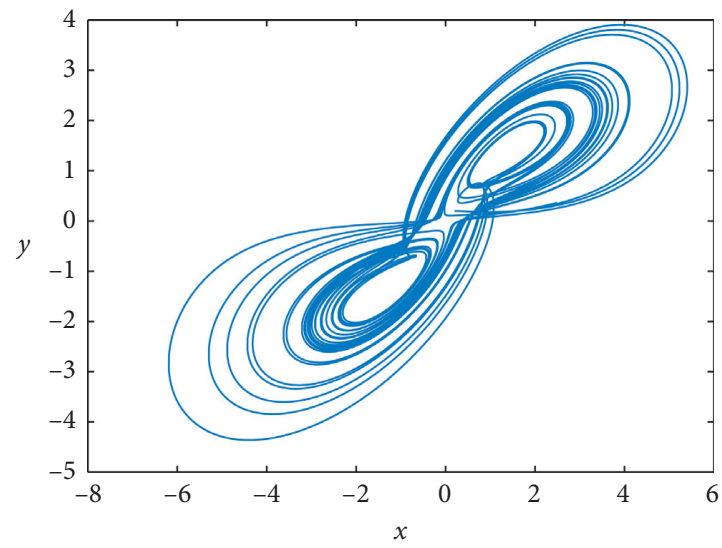

(d)

Figure 16: Phase portraits with system (54)-(56). 


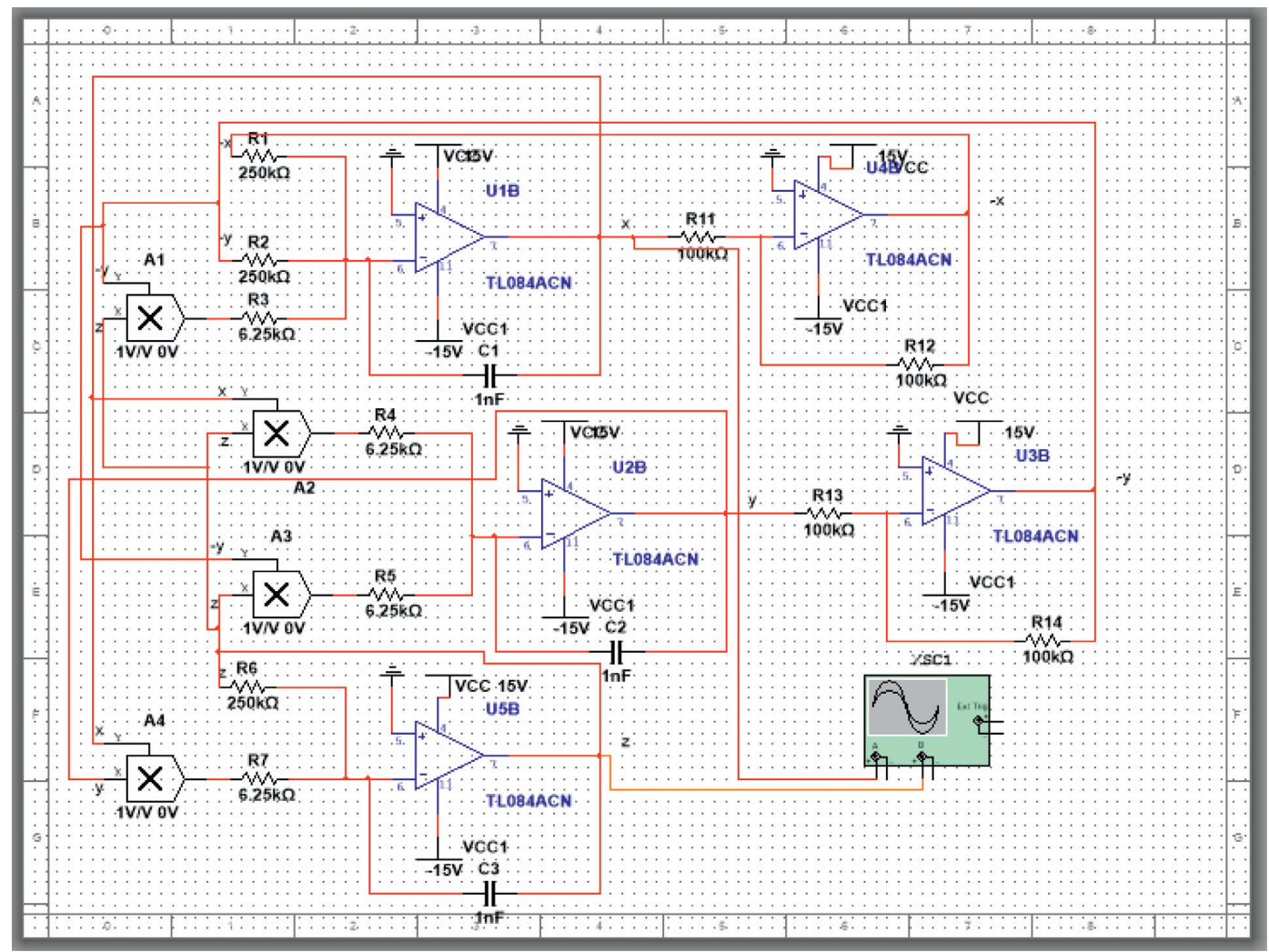

FIGURE 17: Circuit schematic for system (57)-(59).

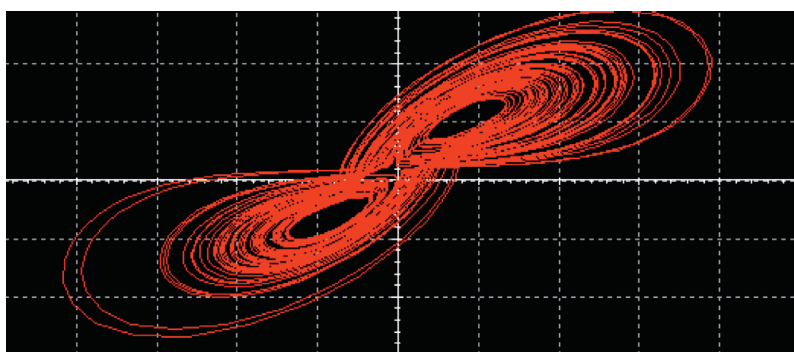

Figure 18: $(x, y)$ space portrait of the Multisim simulation of equations (54)-(56).

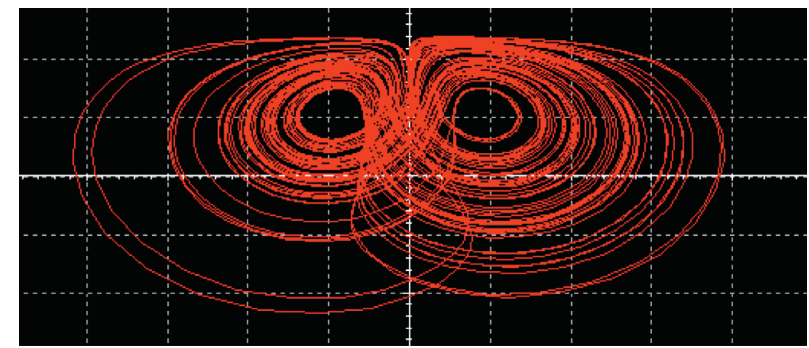

FIGURE 19: $(x, z)$ space portrait of the Multisim simulation of equations (54)-(56).

associated to the initial conditions $(0.2,0.2,0.2)$ (blue) and $(0.5,0.2,0.2)$ (red) versus the variation of the parameter $a$ is given in the following figure.

The bifurcation diagram presented in Figure 25 can be interpreted as with the condition $(0.2,0.2,0.2)$, the chaotic region does not change, and with $(0.5,0.2,0.2)$, the new chaotic region is also the interval $(0.7,5)$. In bifurcation diagrams, see Figures 21, 23, and 25, the general observation is that the chaotic regions do not significantly change when the parameter $a$ varies. We also observe that the chaotic 


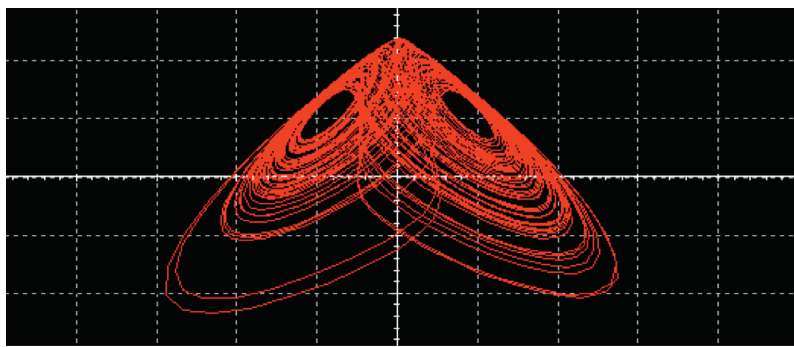

Figure 20: $(y, z)$ space portrait of the Multisim simulation of equations (54)-(56).

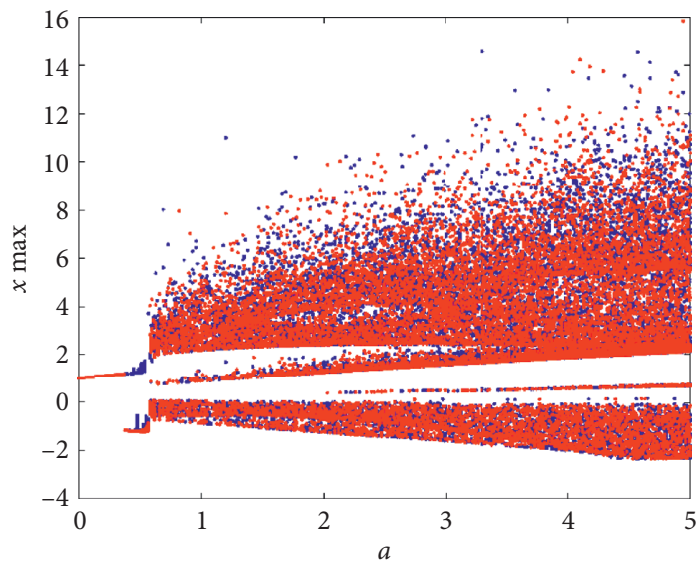

Figure 21: Bifurcation diagram versus the parameter $a$ at $(0.2,0.2,0.2)$ and $(0.2,0.2,0.5)$.

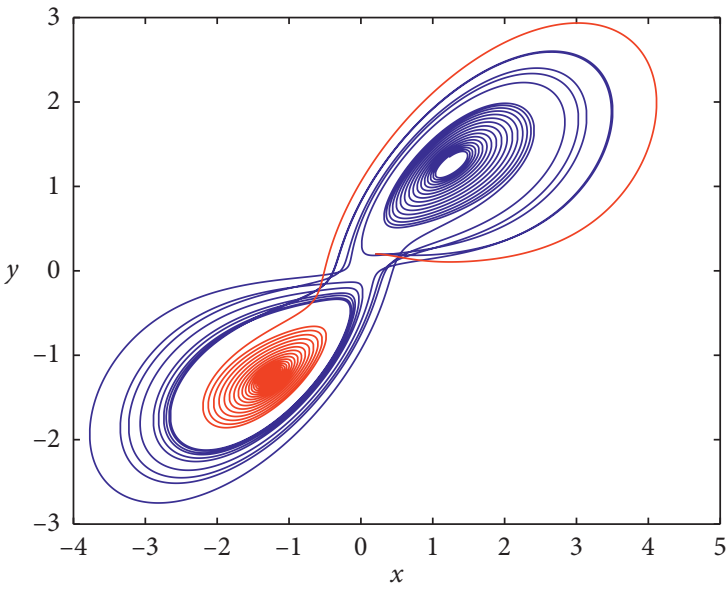

(a)

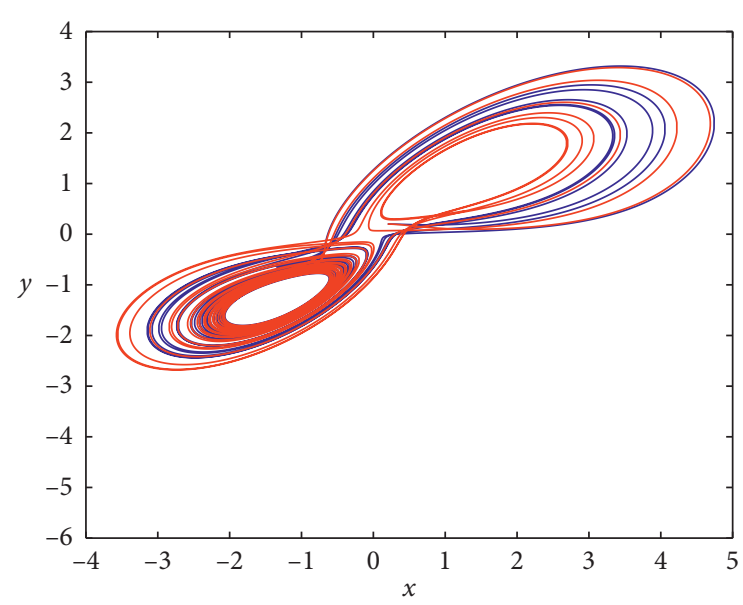

(b)

Figure 22: (a) Portrait at $a=0.6$ in the plane $(x, y)$. (b) Portrait at $a=0.6$ in the plane $(x, y)$. 


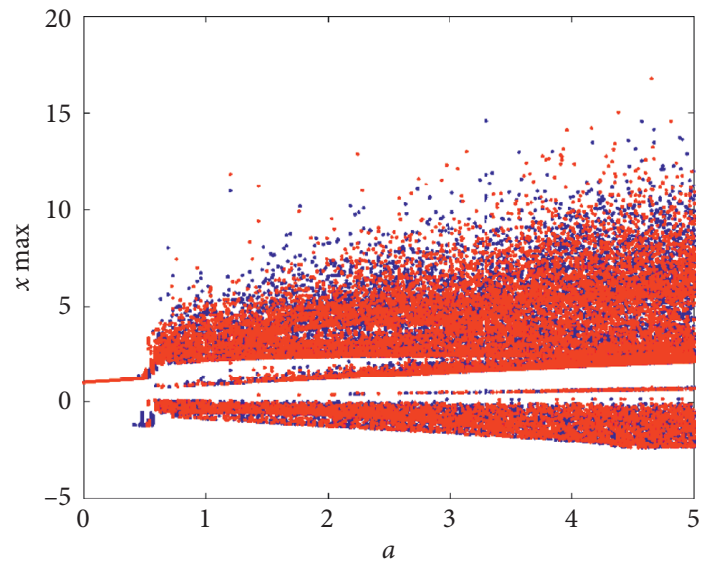

FIGURE 23: Bifurcation diagram versus the parameter $a$ at $(0.2,0.2,0.2)$ and $(0.2,0.5,0.2)$.

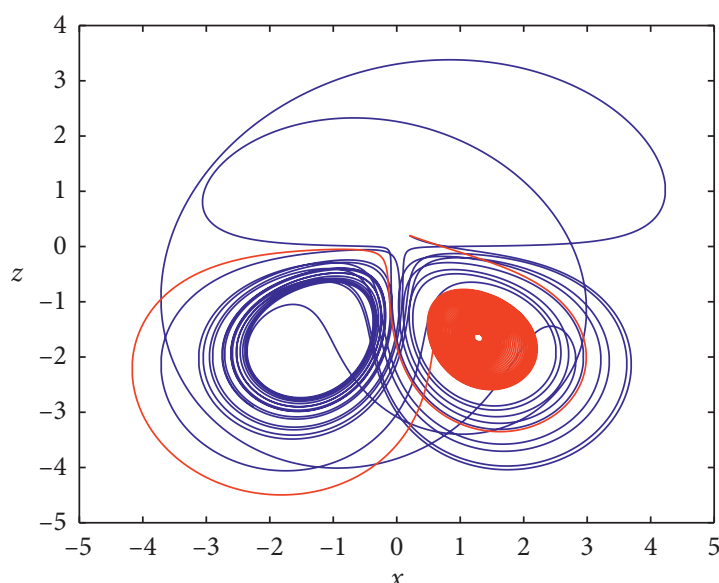

(a)

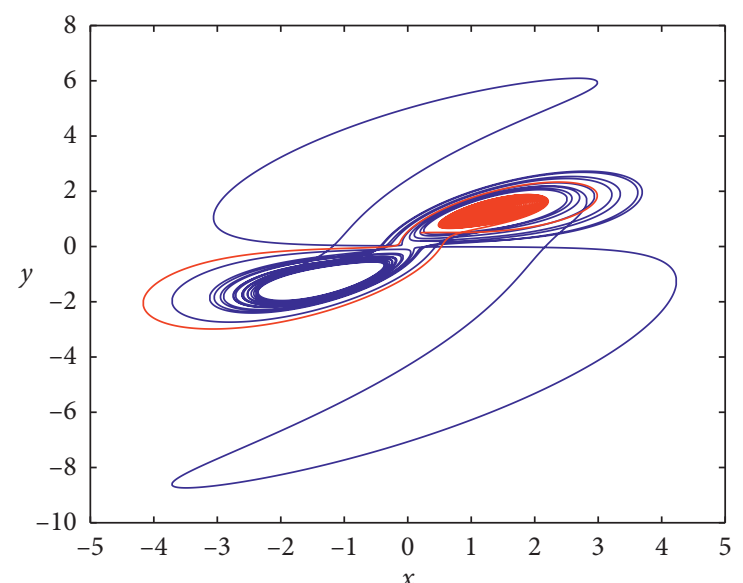

(b)

Figure 24: (a) Portrait at $a=0.65$ in the plane $(x, z)$. (b) Portrait at $a=0.65$ in the plane $(x, y)$.

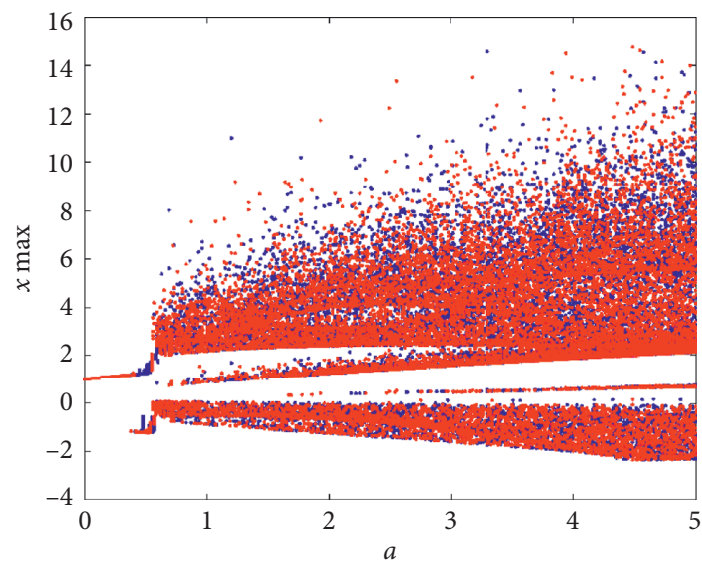

Figure 25: Bifurcation diagram versus the parameter $a$ at $(0.2,0.2,0.2)$ and $(0.5,0.2,0.2)$. 
region $(0.7,5)$ is conserved for all new conditions $(0.2,0.2,0.5),(0.2,0.5,0.2)$, and $(0.5,0.2,0.2)$. Thus, the small variation in the starting conditions does not affect the chaotic region of our present model.

\section{Conclusion}

In this paper, we have proposed a new system of differential equations that exhibit chaotic behavior and then studied with the Caputo fractional derivative. We have discussed the nature of the chaos for different orders of the fractional derivative using Lyapunov exponents. It is noticed that the fractional-order system considered in this paper has chaotic behavior when the order of the fractional derivative is into $(0.9,1)$. The small variation of the model's parameters has been studied using bifurcation diagrams, which play an essential role in the chaos characterization. We also find that the initial condition's choice is crucial because our fractional-order system is very sensitive to the initial conditions. The investigations related to the Lyapunov exponents in the context of the fractional derivative are an open problem. The incommensurate fractional-order chaotic system can be investigated for future work. For example, how the phase portraits can be obtained in the context of incommensurate order. How can the Lyapunov exponents be calculated for a fractional-order chaotic system with incommensurate order? What about the variations of the parameters? What will be the impact of the different fractional-order derivatives?

\section{Data Availability}

No data were used to support this study.

\section{Conflicts of Interest}

The author declares that there are no conflicts of interest.

\section{References}

[1] M. Diouf and N. Sene, "Analysis of the financial chaotic model with the fractional derivative operator," Complexity, vol. 2020, Article ID 9845031, 14 pages, 2020.

[2] L. F. Ávalos-Ruiz, C. J. Zúñiga-Aguilar, J. F. Gómez-Aguilar, R. F. Escobar-Jiménez, and H. M. Romero-Ugalde, "FPGA implementation and control of chaotic systems involving the variable-order fractional operator with Mittag-Leffler law," Chaos, Solitons \& Fractals, vol. 115, pp. 177-189, 2018.

[3] A. Akgul, "Chaotic oscillator based on fractional order memcapacitor," Journal of Circuits, Systems and Computers, vol. 28, no. 14, Article ID 1950239, 2019.

[4] Z. Wei, I. Moroz, J. C. Sprott, A. Akgul, and W. Zhang, "Hidden hyperchaos and electronic circuit application in a 5D self-exciting homopolar disc dynamo," Chaos, vol. 27, p. 033101, Article ID 033101, 2017.

[5] M.-F. Danca, "Lyapunov exponents of a discontinuous 4D hyperchaotic system of integer or fractional order," Entropy, vol. 20 , no. 5 , p. $337,2018$.

[6] F. Mansal and N. Sene, "Analysis of fractional fishery model with reserve area in the context of time-fractional order derivative," Chaos, Solitons \& Fractals, vol. 140, Article ID 110200, 2020.
[7] N. Sene, B. Sène, S. N. Ndiaye, and A. Traoré, "Novel approaches for getting the solution of the fractional blackscholes equation described by Mittag-Leffler fractional derivative," Discrete Dynamics in Nature and Society, vol. 2020, Article ID 8047347, 11 pages, 2020.

[8] M. Yavuz and N. Sene, "Stability analysis and numerical computation of the fractional predator-prey model with the harvesting rate," Fractal and Fractional, vol. 4, no. 3, p. 35, 2020.

[9] S. K. Mishra, M. Gupta, and D. K. Upadhyay, "Fractional derivative of logarithmic function and its applications as multipurpose ASP circuit," Analog Integrated Circuits and Signal Processing, vol. 100, no. 2, pp. 377-387, 2019.

[10] S. K. Mishra, M. Gupta, and D. K. Upadhyay, "Design and implementation of DDCC-based fractional-order oscillator," International Journal of Electronics, vol. 106, no. 4, pp. 581598, 2019.

[11] J. Munoz-Pacheco, E. Zambrano-Serrano, C. Volos, S. Jafari, J. Kengne, and K. Rajagopal, "A new fractional-order chaotic system with different families of hidden and self-excited attractors," Entropy, vol. 20, no. 8, p. 564, 2018.

[12] K. Rajagopal, L. Guessas, A. Karthikeyan, A. Srinivasan, and G. Adam, "Fractional order memristor no equilibrium chaotic system with its adaptive sliding mode synchronization and genetically optimized fractional order PID synchronization," Complexity, vol. 2017, Article ID 189261, , 2017.

[13] J. Gómez-Aguilar, D. Baleanu, and F. Tchier, "Chaotic attractors with fractional conformable derivatives in the Liouville-Caputo sense and its dynamical behaviors," Entropy, vol. 20, no. 5, p. 384, 2018.

[14] J. F. Gómez-Aguilar, K. Ali Abro, O. Kolebaje, and A. Yildirim, "Chaos in a calcium oscillation model via Atangana-Baleanu operator with strong memory," The European Physical Journal Plus, vol. 134, no. 4, p. 140, 2019.

[15] A. Atangana and J. F. Gomez-Aguilar, "Hyperchaotic behaviour obtained via a nonlocal operator with exponential decay and Mittag-Leffler laws," Chaos, Solitons and Fractals, vol. 102, pp. 285-294, 2017.

[16] C. Li, I. Pehlivan, J. Sprott, and A. Akgul, "A novel four-wing strange attractor born in bistability," IEICE Electronics Express, vol. 12, no. 4, pp. 1-12, 2015.

[17] K. Rajagopal, A. Durdu, S. Jafari, Y. Uyaroglu, A. Karthikeyan, and A. Akgul, "Multiscroll chaotic system with sigmoid nonlinearity and its fractional order form with synchronization application," International Journal of Non-Linear Mechanics, vol. 116, pp. 262-272, 2019.

[18] A. Akgul, C. Arslan, and B. Aricioglu, "Design of an interface for random number generators based on integer and fractional order chaotic systems," Chaos Theory and Applications, vol. 1, no. 1, pp. 1-18, 2019.

[19] A. Akgul, C. Li, and I. Pehlivan, "Amplitude control analysis of a four-wing chaotic attractor, its electronic circuit designs and microcontroller-based random number generator," Journal of Circuits, Systems, and Computers, vol. 26, no. 12, Article ID 1750190, 2017.

[20] A. A. Kilbas, H. M. Srivastava, and J. J. Trujillo, Theory and Applications of Fractional Differential Equations, NorthHolland Mathematics Studies, Elsevier, Amsterdam, The Netherlands, 2006

[21] I. Podlubny, Fractional Differential Equations, Mathematics in Science and Engineering, Academic Press, New York, NY, USA, 1999.

[22] J. Fahd, T. Abdeljawad, and D. Baleanu, "On the generalized fractional derivatives and their Caputo modification," Journal 
of Nonlinear Sciences and Applications, vol. 10, pp. 2607-2619, 2017.

[23] R. Garrappa, "Numerical solution of fractional differential equations: a survey and a software tutorial," Mathematics, vol. 6 , no. 2 , p. 16, 2018.

[24] J. L. Echenausía-Monroy, H. E. Gilardi-Velázquez, R. JaimesReátegui, V. Aboites, and G. Huerta-Cuellar, "A physical interpretation of fractional-order-derivatives in a jerk system: electronic approach," Communications in Nonlinear Science and Numerical Simulation, vol. 90, Article ID 105413, 2020.

[25] M. Borah, P. Roy, and B. K. Roy, "Synchronisation control of a novel fractional-order chaotic system with hidden attractor," in Proceedings of the 2016 IEEE Students 'Technology Symposium', Kharagpur, India, September 2016.

[26] S. K. Mishra, D. K. Upadhyay, and M. Gupta, "An approach to improve the performance of fractional-order sinusoidal oscillators," Chaos, Solitons \& Fractals, vol. 116, pp. 126-135, 2018. 